\title{
Matrix Product Unitaries: Structure, Symmetries, and Topological Invariants
}

\author{
J. Ignacio Cirac, ${ }^{1}$ David Perez-Garcia,,${ }^{2,3}$ Norbert Schuch, ${ }^{1}$ and Frank Verstraete ${ }^{4,5}$ \\ ${ }^{1}$ Max-Planck-Institut für Quantenoptik, Hans-Kopfermann-Str. 1, 85748 Garching, Germany \\ ${ }^{2}$ Departamento de Anlisis Matemtico, Universidad Complutense de Madrid, Plaza de Ciencias 3, 28040 Madrid, Spain \\ ${ }^{3}$ ICMAT, Nicolas Cabrera, Campus de Cantoblanco, 28049 Madrid, Spain \\ ${ }^{4}$ Department of Physics and Astronomy, Ghent University, Krijgslaan 281, S9, 9000 Gent, Belgium \\ ${ }^{5}$ Vienna Center for Quantum Science and Technology, Faculty of Physics, \\ University of Vienna, Boltzmanngasse 5, 1090 Vienna, Austria
}

\begin{abstract}
Matrix Product Vectors form the appropriate framework to study and classify one-dimensional quantum systems. In this work, we develop the structure theory of Matrix Product Unitary operators (MPUs) which appear e.g. in the description of time evolutions of one-dimensional systems. We prove that all MPUs have a strict causal cone, making them Quantum Cellular Automata (QCAs), and derive a canonical form for MPUs which relates different MPU representations of the same unitary through a local gauge. We use this canonical form to prove an Index Theorem for MPUs which gives the precise conditions under which two MPUs are adiabatically connected, providing an alternative derivation to that of Ref. 1 for QCAs. We also discuss the effect of symmetries on the MPU classification. In particular, we characterize the tensors corresponding to MPU that are invariant under conjugation, time reversal, or transposition. In the first case, we give a full characterization of all equivalence classes. Finally, we give several examples of MPU possessing different symmetries.
\end{abstract}

\section{INTRODUCTION}

Strongly correlated quantum systems display a wide range of unconventional phenomena which emerge from the close interplay between their locality structure and the presence of strong interactions. Tensor Networks accurately capture the complex correlations present in such systems while at the same time preserving their locality structure, and thus form a versatile framework for their analytical characterization and numerical study. In one spatial dimension, this gives rise to a Matrix Product Vector (MPV) structure which appears in a wide range of scenarios, encompassing both genuinely onedimensional systems and boundaries of two-dimensional systems. Specifically, Matrix Product States (MPS) allow for the efficient description of low-energy states of one-dimensional systems [2, 3]. Matrix Product Density Operators (MPDOs) [4, 5] describe thermal states in one dimension as well as boundaries of gapped twodimensional systems [6], whereas topological order and anyonic excitations can be characterized using Matrix Product Operator Algebras [7, 8. The evolution under one-dimensional Hamiltonians and Floquet operators [9], as well as the effect of bulk symmetries on boundaries of two-dimensional systems [10], can be described in terms of Matrix Product Unitaries (MPUs).

A central application of MPVs is the study and classification of phases of strongly correlated systems, this is, equivalence classes under smooth deformations, based on discrete invariants labelling the MPVs of interest. The precise notion of equivalence depends on the physical context and the constraints imposed, where overall two scenarios need to be distinguished: For MPVs describing genuinely one-dimensional systems, the appropriate notion of equivalence characterizes whether it is possible to smoothly deform an MPV into another one while keeping certain properties. In contrast, for MPV characterizing boundaries of two-dimensional systems a smooth interpolation of the two-dimensional bulk is required, to which end it rather matters whether one can locally combine the boundary MPVs into a joint MPV with the same structure.

One of the most important achievements of MPS has been the full classification of zero-temperature phases of gapped one-dimensional quantum systems, based on previously derived canonical forms of MPS and their connection to parent Hamiltonians [11. It has been proven that without symmetries, any two MPS can be connected along a path corresponding to a gapped Hamiltonian, while in the presence of symmetries, the inequivalent phases are characterized by the symmetry action at the boundary (this is, on the entanglement), labelled by group cohomology and generalizations thereof 12,14$]$. Invariants characterizing different phases have also been identified in a range of other contexts: Local symmetries of $2 \mathrm{D}$ phases are represented at the boundary by MPUs, which in turn are labelled by the third group cohomology [10, MPOs describing topologically ordered wavefunctions locally can be characterized in terms of tensor fusion categories [7, 8, and scale-invariant MPDOs appearing at the boundary of renormalization fixed points exhibit an algebraic structure given by fusion categories [15. The central tool for all these results has been the identification of suitable canonical forms for the corresponding MPVs, which allow to relate different MPV descriptions of the same state locally, and to classify phases through the algebraic structure of the underlying gauge transforms [11, 15].

In this paper, we develop a structure theory of Matrix Product Unitaries (MPU) for one-dimensional systems. Specifically, we provide a characterization of the general structure of arbitrary MPUs, and use this to derive a canonical form for MPUs which relates different representations of the same MPU through a local gauge. We 
find that all MPUs are, in fact, Quantum Cellular Automata (QCAs) [16, this is, they propagate information strictly by a finite distance only. Based on our standard form, we define an index which measures the net quantum information flow through the MPU, and give a Matrix Product based derivation of the Index Theorem of Gross et al. [1] which shows that the index precisely labels equivalence classes of MPUs under smooth deformations. We also characterize the tensors generating MPUs with one of the following symmetries: conjugation, time reversal, and transposition. For the case of conjugation, we fully classify the corresponding equivalence classes, and for all symmetries, we connect our results with the characterization of symmetry protected topological phases in MPS. We conclude with some relevant examples of MPUs possessing the different symmetries.

Recently, MPUs have been used to describe the time evolution operator in the study of Floquet phases, based on the intuition that the Matrix Product structure captures the time evolution under local Hamiltonians [9]; with the additional assumption that these MPUs describe QCAs, this allowed to use the Index Theorem of Ref. [1] to classify certain Floquet phases. Our result removes the need for this additional assumption by proving its general validity, and gives a direct way of classifying MPUs based on their standard form, providing the tools for generalizations to systems with symmetries in the MPV formalism. Let us note that the effect of symmetries on the classification of time evolutions has recently been investigated for one-dimensional random walks of single particles [17, which in the absence of symmetries share many common features with QCAs. We believe that the structure theory of MPUs developed in this work will allow to generalize these results to the domain of interacting systems [18.

This paper is organized as follow: In Section II we review some of the basic properties of MPVs that will be used along the paper. In Section III we derive the general structure of MPUs and characterize them with a Standard Form. In Section IV we introduce the Index [1] and prove its robustness. Section V contains the characterization of tensors corresponding to MPUs with different symmetries. Section VI analyzes the equivalences among them, and Section VII includes some relevant examples. The connection between MPUs and QCAs is given in the Appendix.

\section{BASICS IN MATRIX PRODUCT VECTORS}

In this section we will recall the basic definitions and results about Matrix Product Vectors from Ref. [15].

Let us consider a Hilbert space of dimension $d_{0}, H_{d_{0}}$, a basis $\left\{e_{n}\right\}_{n=1}^{d_{0}}$, and a tensor, $\mathcal{A}$, of complex coefficients $A_{\alpha, \beta}^{n}$, with $n=1, \ldots, d_{0}$ and $\alpha, \beta=1, \ldots, D$. The tensor defines a family of vectors, $\left\{V^{(N)} \in H_{d}^{\otimes N}\right\}_{N=1}^{\infty}$, as

$$
V^{(N)}=\sum_{n_{1}, \ldots, n_{N}=1}^{d_{0}} c_{n_{1}, \ldots, n_{N}} e_{n_{1}} \otimes \ldots \otimes e_{n_{N}}
$$

where

$$
c_{n_{1}, n_{2}, \ldots, n_{N}}=\sum_{\alpha_{1}, \ldots, \alpha_{N}=1}^{D} \prod_{k=1}^{N} A_{\alpha_{k}, \alpha_{k+1}}^{n_{k}}
$$

are complex coefficients and with the identification $\alpha_{N+1}=\alpha_{1}$. We call the $V^{(N)}$ matrix product vectors (MPV), and we say that $\mathcal{A}$ generates the MPV, where the $\alpha$ 's and $n$ are the bond (or auxiliary) and physical indices, respectively, and $D$ and $d_{0}$ their corresponding dimensions. We will use the same graphical notation as in [15]. First, we represent the tensor $\mathcal{A}$ as

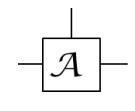

where the horizontal (vertical) lines represent the bond (physical) indices. We will represent the MPV

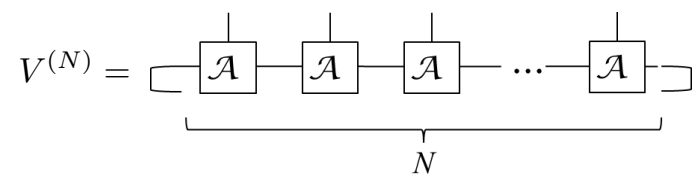

Here, the lines that join different tensors indicate that the corresponding indices are contracted, while the vertical lines (which are open) represent the physical indices. The curvy lines at the end indicate that the last and first tensors are also contracted. This graphic means that the coefficients of $V^{(N)}$ can be determined by assigning values to the physical (open) indices, multiplying the matrices and then taking the trace.

Definition II.1. The transfer operator is defined as

$$
E=\sum_{n=1}^{D} A^{n} \otimes \bar{A}^{n}
$$

where $A^{n}$ are matrices with elements $A_{\alpha, \beta}^{n}$, and the bar denotes complex conjugate. We will denote by $\lambda_{E}$ the spectral radius of $E$, i.e. the eigenvalue with largest $a b$ solute value.

As the MPV generated by two different tensors may coincide, it is useful to define a canonical form:

Definition II.2. We say that a tensor $\mathcal{A}$ generating $M P V$ is in canonical form (CF) if: (i) the matrices are of the form $A^{n}=\oplus_{k=1}^{r} \mu_{k} A_{k}^{n}$, where $\mu_{k} \in \mathbb{C}$ and the spectral radius of the transfer matrix, $E_{k}$, associated to $A_{k}^{n}$ is equal to one; (ii) for all $k$, there exists no projector, $P_{k}$, such that $A_{k}^{n} P_{k}=P_{k} A_{k}^{n} P_{k}$ for all $n$, or $P_{k} A_{k}^{n}=P_{k} A_{k}^{n} P_{k}$ for all $n$. 
This basically means that the matrices $A^{n}$ are written in a block-diagonal form and there is no way of making the blocks smaller. Furthermore, if two tensors, $\mathcal{A}, \mathcal{B}$, are related to each other by a gauge transformation $(\mathcal{B}=$ $X \mathcal{A} X^{-1}$ ) then they generate the same MPV. Using this fact, one can show that for any tensor it is always possible to find another one that generates the same MPV and is in $\mathrm{CF}$.

Definition II.3. We say that a tensor generating MPV is normal if it is in CF, has a single block $(r=1)$, and its associated transfer matrix has a unique eigenvalue of magnitude (and value) equal to its spectral radius, which is equal to one.

Note that the transfer matrix $E_{k}$ associated to $A_{k}^{n}$ in $\mathrm{CF}$ may still have several eigenvalues of magnitude equal to their spectral radius (the so-called $p$-periodic states). For normal tensors, however, this is not the case for its single block. Furthermore, its transfer matrix has no Jordan block corresponding to the eigenvalue one. Moreover, the condition that the spectral radius is equal to one can always be met by multiplying the tensor by a constant.

Let us denote by $\Phi$ and $\rho$ the left and right eigenvectors of $E$ corresponding to the eigenvalue 1 of a normal tensor.

Definition II.4. For normal tensors, we say that $\mathcal{A}$, generating $M P V$, is in Canonical Form II (CFII) if

$$
\begin{aligned}
& \left(\Phi \mid=\sum_{n=1}^{D}(n, n \mid,\right. \\
& \left.\mid \rho)=\sum_{n=1}^{D} \rho_{n} \mid n, n\right),
\end{aligned}
$$

where $\rho_{n}>0$ and $(\Phi \mid \rho)=1$.

Since $\Phi$ and $\rho$ are rank-2 tensors, they can be considered as $D \times D$ matrices, or as vectors in $H_{D} \otimes H_{D}$. We will use those considerations depending on the context. Graphically,

$$
E=\stackrel{-\overline{\mathcal{A}}-}{-1}
$$

and

$$
\frac{\sqrt{\overline{\mathcal{A}}}}{\sqrt{\mathcal{A}}-}=\left[\text { and } \frac{-\overline{\mathcal{A}}}{-\bar{A}}=?\right.
$$

Here, the tensor with the circle represents the right eigenvector $\rho$.

For a normal tensor it is always possible to find a gauge transformation defining a new normal tensor that is in CFII and generates the same MPV. Another concept that we will use later on is that of blocking.
Definition II.5. Given $\mathcal{A}$ generating $M P V$, we denote by $\mathcal{A}_{k}$ the tensor obtained after blocking $k$ tensors

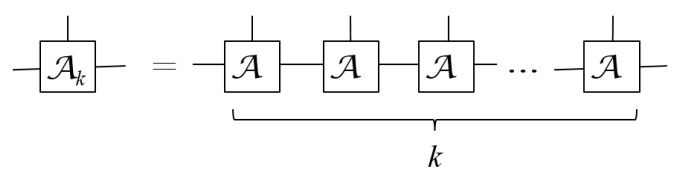

In (9), the vertical indices have been combined into a single one. Note that $d_{k}=d_{0}^{k}$ and $D$ are the dimension of the local physical Hilbert space and bond dimension corresponding to $\mathcal{A}_{k}$, respectively.

In the following, we will transition to Matrix Product Operators, for which $H_{d_{0}}$ will describe the space of operators acting on a Hilbert space $H_{d}$ of dimension $d$, and the MPV will thus represent operators acting on $H_{d}^{\otimes N}$. In the case where these operators are unitary, we will term them Matrix Product Unitaries (MPU) [10]. In order to emphasize that the tensor generates MPU, we will denote it by $\mathcal{U}$ and the corresponding MPU by $U^{(N)}$.

\section{STUCTURE OF MPU}

The aim of this section is to characterize when a tensor $\mathcal{U}$ generates translationally invariant MPU, i.e., when

$$
U^{(N)}=[\underbrace{\frac{1}{u}-\underbrace{u}_{1}-\underbrace{\frac{1}{u}}_{1}-\frac{1}{u}-\ldots-\frac{1}{u}]}_{N}
$$

is a unitary operator for $N=2,3 \ldots$ We will also introduce a standard form for such tensors and a fundamental theorem which will allow us to relate tensors generating the same family of MPU.

As explained in the previous section, unless we specify the contrary, wlog we can assume that the tensor $\mathcal{U}$ is in CF. We will denote by $\overline{\mathcal{U}}$ the tensor obtained from $\mathcal{U}$ by transposing the physical indices and conjugating all coefficients, and use the graphical notation

$$
-\frac{1}{u}-\stackrel{+!}{u}
$$

The unitarity of the MPU is equivalent to $U^{(N) \dagger} U^{(N)}=$ $\mathbb{1}^{\otimes N}$. Graphically,

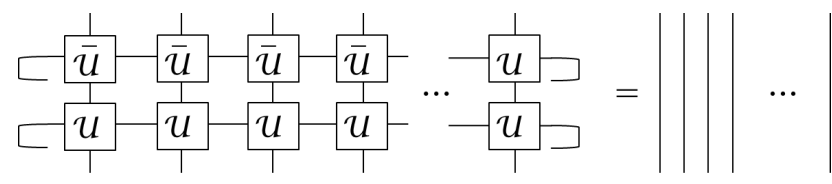

We also define the transfer matrix of $\frac{1}{\sqrt{d}} \mathcal{U}$

$$
E=\frac{1}{d} \underset{\substack{u \\ u}}{-\sqrt{u}}
$$


Let us start with the following simple observation:

Proposition III.1. E has just one nonzero eigenvalue, which is equal to one, and $\frac{1}{\sqrt{d}} \mathcal{U}$ is a normal tensor.

Proof. Since $U^{(N)}$ is unitary for all $N>1$, then $\operatorname{tr}\left(E^{N}\right)=$ $\frac{1}{d^{N}} \operatorname{tr}\left(U^{(N)} U^{(N) \dagger}\right)=1$ for all $N>1$. Thus, the spectrum of $E$ contains a one and the rest of the eigenvalues vanish, as it trivially follows from Lemma A.5 in [15. This is incompatible with having more than one block in the CF. Furthermore, the transfer matrix does not have more than one eigenvalue of magnitude equal to one, so that the tensor must be normal.

As a consequence of this observation, wlog we can assume that $\frac{1}{\sqrt{d}} \mathcal{U}$ is in CFII. Following the Definition II.5 we will denote by $\mathcal{U}_{k}$ the tensor corresponding to blocking $\mathcal{U} k$ times. Note also that this tensor is also normal and in CFII.

\section{A. Characterization of MPU via simple tensors}

We will consider a special kind of tensors generating MPU, which have a peculiar property, from which we can derive their general structure. Then we will show that by blocking a final number of times, any tensor generating MPU develops such a property.

Definition III.2. We say that a tensor $\mathcal{U}$ is simple if there exist two tensors, $a$ and $b$, such that
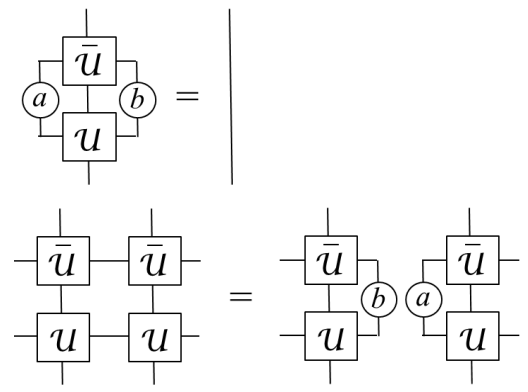

Now we show that by blocking, any tensor generating MPU becomes simple.

Proposition III.3. (i) Any simple tensor generates $M P U$. (ii) For any tensor generating $M P U, \mathcal{U}$, there exists some $k \leq D^{4}$ such that $\mathcal{U}_{k}$ is simple, where tensors $a, b$ are the left and right fixed points, $\Phi$ and $\rho$, of the transfer operator $E$, respectively.

Proof. (i) One can just apply sequentially (14) to $U^{(N) \dagger} U^{(N)}$ to obtain 12 so that $U^{(N)}$ is indeed unitary for $N>1$.

(ii) Given $\mathcal{U}$ generating MPU, we can always write

$$
\frac{\frac{1}{\bar{u}}}{-\frac{1}{u}}=E \otimes \mathbb{1}+\sum_{\alpha} S_{\alpha} \otimes \sigma_{\alpha}
$$

Here, the first part of the tensor products acts on the auxiliary indices, whereas the second part on the physical ones. Furthermore $E$ is the transfer operator defined in (13) whereas the $\sigma_{\alpha}$ are traceless operators.

By proposition III.1, $E$ has a unique non-zero eigenvalue equal to 1 . Since $\mathcal{U}$ is in CFII, its associated left and right eigenvectors, $\Phi$ and $\rho$, are given by (6). Graphically, we have [cf. (7)]

$$
\frac{1}{d \bar{u}} \frac{\sqrt{u}}{\sqrt{u}}=\left[\begin{array}{l}
-\bar{u} \\
\frac{u}{u}
\end{array}=?\right.
$$

where the circle represents the right eigenvector, $\rho$. Furthermore, there is no Jordan block associated to this eigenvalue, whereas there may be one or several Jordan blocks associated to the zero eigenvalues. If we denote by $J<D^{2}$ the largest size of all Jordan blocks of $E$, let us block now $J$ sites and consider $\mathcal{U}_{J}$. Given that this tensor itself generates MPU and is in CFII, we can use for it the decomposition 15 , where we will denote by $E^{\prime}$ and $S_{\alpha}^{\prime}$ the new matrices. We have $\left.E^{\prime}=\mid \rho\right)(\Phi \mid$, since after blocking all Jordan blocks will disappear. Taking into account 12 we conclude that the trace of any product of operators containing one or more $S_{\alpha}^{\prime}$ vanishes. This implies that

$$
E^{\prime} S_{\alpha_{1}}^{\prime} \ldots S_{\alpha_{m}}^{\prime} E^{\prime}=\operatorname{tr}\left(E^{\prime} S_{\alpha_{1}}^{\prime} \ldots S_{\alpha_{m}}^{\prime}\right)=0
$$

for any $m \geq 1$. Furthermore, any element, $S$, in the algebra generated by the $S_{\alpha}^{\prime}$ must have zero eigenvalues since $\operatorname{tr}\left(S^{N}\right)=0$ for all $N$, and thus they are nilpotent. It follows then from a result of Nagata and Higman [19, 20, improved later by Razmyslov [21], that there exists some $J^{\prime}<D^{2}$ such that

$$
S_{\alpha_{1}}^{\prime} \ldots S_{\alpha_{J^{\prime}}}^{\prime}=0
$$

for any set of $\alpha$ 's (see 22 for a review about this type of questions). Let us now block again, $J^{\prime}$ times, so that we consider $\mathcal{U}_{J J^{\prime}}$. Again, we can use the decomposition (15) and denote by $E^{\prime \prime}=E^{\prime}$ and $S_{\alpha}^{\prime \prime}$ the new matrices. The latter can have one of the following three types of structures:

$$
\begin{array}{ll}
\text { type1: } & S_{\alpha}^{\prime \prime}=E^{\prime}\left[\prod_{k} S_{\alpha_{k}}^{\prime}\right], \\
\text { type2: } & S_{\alpha}^{\prime \prime}=\left[\prod_{k} S_{\alpha_{k}}^{\prime}\right] E^{\prime}, \\
\text { type3: } & S_{\alpha}^{\prime \prime}=\left[\prod_{k} S_{\alpha_{k}}^{\prime}\right] E^{\prime}\left[\prod_{\ell} S_{\alpha_{\ell}}^{\prime}\right] .
\end{array}
$$

The reason is that whenever two non-consecutive $E^{\prime}$ appear, the operator vanishes due to (17), and the one where no $E^{\prime}$ appears vanishes as well due to (18).

Given the fact that $E^{\prime \prime} S^{\prime \prime} E^{\prime \prime}=0$ and that $E^{\prime \prime} E^{\prime \prime}=E^{\prime \prime}$, we immediately obtain $14 \mathrm{a}$ for $\mathcal{U}_{J J^{\prime}}$ with $a=\Phi$ and 
$b=\rho$. Now, let us consider the lhs of $14 \mathrm{~b}$. When we replace decomposition (15) in each of the columns, we will have products of the form $A_{1} A_{2}$, where $A_{1,2}$ will be either $E^{\prime \prime}$ or one of the $S_{\alpha}^{\prime \prime}$. We will show now that $A_{1} A_{2}=A_{1} E^{\prime \prime} A_{2}$, which will immediately imply $14 \mathrm{~b}$, with $a=\Phi$ and $b=\rho$. This is obvious whenever $A_{1}$ or $A_{2}$ is equal to $E^{\prime \prime}$. Thus, we just have to show that $S_{\alpha}^{\prime \prime} S_{\beta}^{\prime \prime}=S_{\alpha}^{\prime \prime} E^{\prime \prime} S_{\beta}^{\prime \prime}$. But this follows automatically from (19), since this product vanishes unless $S_{\alpha}^{\prime \prime}$ is of type 2 and $S_{\beta}^{\prime \prime}$ is of type 1 . In that case, we can write $S_{\alpha}^{\prime \prime}=S_{\alpha}^{\prime \prime} E^{\prime \prime}$, from which the statement follows. Note that we have blocked $J J^{\prime}<D^{4}$ times.

Corollary III.4. Equation (14a) holds for any tensor $\mathcal{U}$ generating MPU (not necessary simple), where the tensors $a, b$ are the left and right fixed points, $\Phi$ and $\rho$, of the transfer operator $E$.

Proof. Take $J \leq D^{2}$ as in the previous Proposition and consider $U^{(J+1)}$. The LHS of $14 \mathrm{a}$ is simply

$$
\frac{1}{d^{J}} \operatorname{tr}_{1, \ldots, J}\left(U^{(J+1) \dagger} U^{(J+1)}\right),
$$

which is clearly the identity, since $U^{(N)}$ is unitary.

This in turn implies:

Corollary III.5. Let $\mathcal{U}$ be a tensor generating $M P U$. If its blocked tensor $\mathcal{U}_{k}$ is simple, the same is true for all $\mathcal{U}_{k^{\prime}}, k^{\prime} \geq k$.

\section{B. Standard form}

In the previous section we have seen that, starting with a tensor generating MPU in $\mathrm{CF}$, it is enough to block $k<$ $D^{4}$ sites to have a simple tensor. In this subsection we will characterize the minimal such $k$ in terms of the ranks of two matrices associated to $\mathcal{U}$. As a corollary we will obtain an (essentially unique) standard form for simple tensors. Another corollary will show that any MPU is a quantum cellular automaton (QCA) and vice versa.

We consider two different singular value decompositions of a (not necessarily simple) tensor $\mathcal{U}$ generating MPU, corresponding to two different choices of how we combine the indices of $\mathcal{U}$ in order to write it as matrices, $\mathcal{M}_{1}$ and $\mathcal{M}_{2}$, respectively. In the first decomposition, we combine the left auxiliary and down physical index, as well as the other two, whereas in the second, the other way around. Graphically, the separation in two sets of indices is represented by a dashed line:

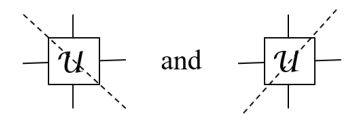

Definition III.6. We denote by $r$ and $\ell$ the rank of $\mathcal{M}_{1,2}$, respectively.
Using a singular value decomposition, we can write

$$
\mathcal{M}_{1,2}=V_{1,2}^{\dagger} D_{1,2} U_{1,2}
$$

where the $U$ 's and $V$ 's are isometries, fulfilling $V_{i} V_{i}^{\dagger}=$ $U_{i} U_{i}^{\dagger}=\mathbb{1}$, and $D_{1,2}$ are diagonal positive matrices, of dimensions $r$ and $\ell$, respectively. We define now matrices $X_{i}$ and $Y_{i}$ such that $\mathcal{M}_{i}=X_{i} Y_{i}$ in terms of those decompositions. We choose $X_{2}=V_{2}^{\dagger}$ and $X_{1}=$ $V_{1}^{\dagger}\left[V_{1}(\mathbb{1} \otimes \rho) V_{1}^{\dagger}\right]^{-1 / 2}$, so that we have

$$
X_{2}^{\dagger} X_{2}=\mathbb{1}, \quad X_{1}^{\dagger}(\mathbb{1} \otimes \rho) X_{1}=\mathbb{1} .
$$

Recall that $\rho$ is a diagonal matrix with coefficients $\rho_{n}$ that appear in the right eigenvector of the transfer matrix (13), see $(16)$. Since $\rho_{n}>0$, the square roots and inverses are well defined. Additionally, defining

$$
\begin{aligned}
& Z_{2}=U_{2}^{\dagger} D_{2}^{-1} \\
& Z_{1}=U_{1}^{\dagger} D_{1}^{-1}\left[V_{1}(\mathbb{1} \otimes \rho) V_{1}^{\dagger}\right]^{-1 / 2}
\end{aligned}
$$

we have

$$
Y_{1} Z_{1}=Y_{2} Z_{2}=\mathbb{1}
$$

and therefore the $Y_{i}$ are invertible.

Graphically, we can identify some tensors with those matrices

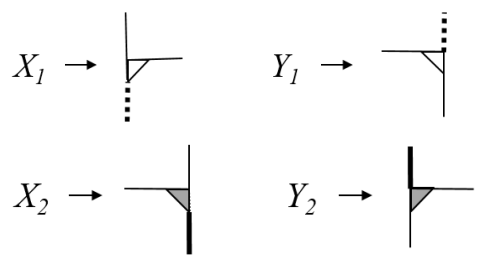

Note that we have represented all those tensors by triangles with the hypotenuse below the triangle. We have also filled the triangles corresponding to the second decomposition, and use thick dotted and solid lines to represent the bonds with dimensions $r$ and $\ell$, respectively. With this notation, we can re-express 23 as

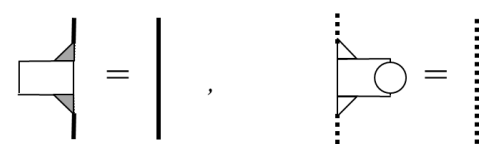

We can thus decompose the tensor $\mathcal{U}$ as

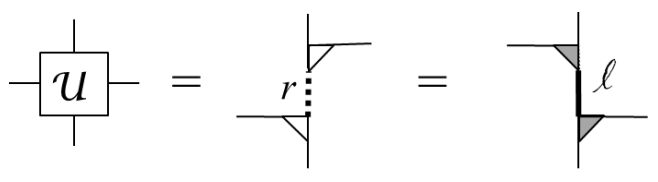

In terms of the previous decompositions, we define

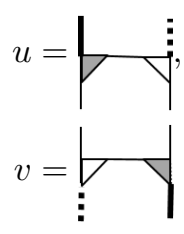


Note that both $u$ and $v^{\dagger}$ map a space of $d^{2}$ dimension onto another one of dimension $r \ell$.

Lemma III.7. For any tensor $\mathcal{U}, u^{\dagger} u=\mathbb{1}$ and hence $r \ell \geq d^{2}$

Proof. Using (27) we have

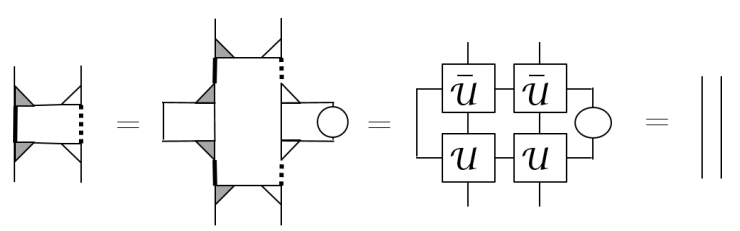

so that $u^{\dagger} u=\mathbb{1}$. For the last equality we have used that choosing $k \leq D^{4}$ such that $\mathcal{U}_{k}$ is simple (see Proposition III.3, the $\mathrm{lh}$ s is equal to $1 / d^{k} \operatorname{tr}_{1, \ldots, k}\left[U^{(k+2) \dagger} U^{(k+2)}\right]=$ $\mathbb{1 1}$.

Note that this lemma implies that $u$ is an isometry. In order for it to be unitary, it is required that $r \ell=d^{2}$. Now, we are in the position of stating the main result of this section:

Theorem III.8. The following are equivalent for a tensor $\mathcal{U}$ generating MPU with the above definitions.

1. $\mathcal{U}$ is simple.

2. $r \ell=d^{2}$.

3. $u$ is unitary.

4. $v$ is unitary.

Proof. $1 \rightarrow 2$ : Let us assume that $\mathcal{U}$ is simple. Using (14) we have

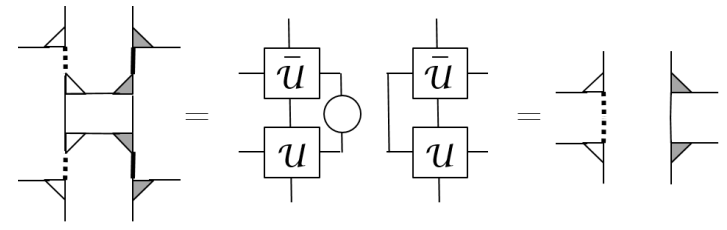

where in the last step we have used (27). Employing (26) we have

$$
\left(Y_{1} \otimes Y_{2}\right)^{\dagger} v^{\dagger} v\left(Y_{1} \otimes Y_{2}\right)=\left(Y_{1} \otimes Y_{2}\right)^{\dagger}\left(Y_{1} \otimes Y_{2}\right)
$$

Conjugating both sides of this equation with $\left(Z_{1} \otimes Z_{2}\right)^{\dagger}$ and $\left(Z_{1} \otimes Z_{2}\right)$ and using 25) we obtain that $v^{\dagger} v=\mathbb{1}$. This implies that $r \ell \leq d^{2}$, which together with Lemma III.7 implies $r \ell=d^{2}$.

$2 \rightarrow 3$ : If we assume now $r \ell=d^{2}$, through Lemma III.7 we immediately get that $u$ is unitary (note that $u^{\dagger} u=\mathbb{1}$ ). $3 \rightarrow 4$ : If $u$ is unitary, then since $U^{(2)}$, which is also unitary, is just the product of $u$ with $v$ translated by one site, we deduce that $v$ is also unitary.

$4 \rightarrow 1$ : Finally, if $v$ is unitary, by Corollary III.4 it is enough to show (14b), which is just the first equality in (31). The fact that $v$ is unitary provides us with the equality between the LHS and the RHS of (31). By inserting (27) in the RHS [as done in [12] ] we obtain the desired equality with the middle term in (31).
Equipped with this theorem, we have a practical way of computing $k_{0}$, namely the smallest $k$ such that $\mathcal{U}_{k}$ is simple. We just have to block and compute $r_{k}$ and $\ell_{k}$ for $k=1,2, \ldots$ until we obtain $r_{k_{0}} \ell_{k_{0}}=d_{k_{0}}^{2}$; as we have shown in the previous subsection, $k_{0} \leq D^{4}$. Additionally, it also gives us a practical way to check if a tensor $\mathcal{U}$ generates MPUs: after blocking and reaching $r_{k_{0}} \ell_{k_{0}}=$ $d_{k_{0}}^{2}$, we just have to check if the resulting tensor is simple. This theorem also shows that by blocking a simple tensor $\mathcal{U}$ two times we have

$$
-\frac{1}{u_{2}}=\frac{\frac{1}{!}}{\frac{u}{u}}
$$

where $u$ is a unitary and $v$, as defined in (29b), too. This will define a standard form for all MPU (after blocking $2 k_{0}$ times).

Definition III.9. Given a simple tensor, $\mathcal{U}$, we define the standard form $(S F)$ of $\mathcal{U}_{2}$ as (33).

Now, we derive the fundamental theorem of MPU, namely the relation between two tensors generating MPU in SF. For that, let us consider two simple tensors, $\mathcal{U}$ and $\mathcal{V}$, generating the same MPU, and with standard forms

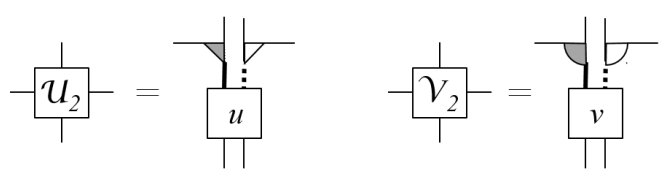

Using the fact that $X_{1}$ is invertible, it is straightforward to prove the following result, which we call the fundamental theorem of MPU:

Theorem III.10 (Fundamental Theorem of MPU). Given two simple tensors, $\mathcal{U}$ and $\mathcal{V}$ with standard forms (34), they generate the same $M P U$, i.e. $U^{(N)}=V^{(N)}$ for all $N$, iff there exist unitaries $x, y$ and $z$, such that

$$
\underset{T=1}{i=}
$$
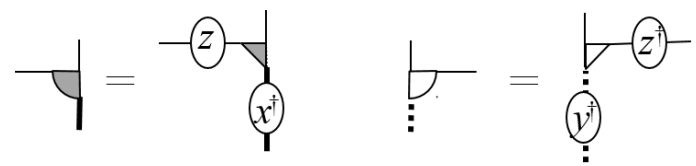

Note that the unitarity of $z$ follows from the fact that both tensors are in CFII [15].

Using this standard form, we can also identify the tensors $X$ and $Y$ corresponding to its singular value decompositions

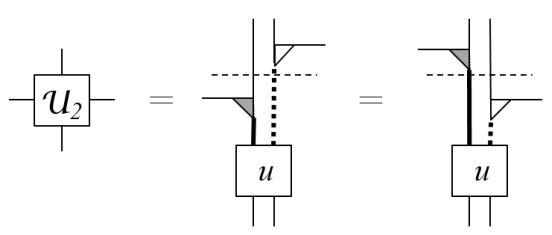


where for the sake of clarity, we have separated with a thin line the two pieces of the singular value decomposition.

We finish this section making the announced identification of MPU's and QCA. We refer to the Appendix for the formal definition of QCA and the proof of the following

Corollary III.11. Any MPU (with finite bond dimension) is a $1 D Q C A$ and viceversa.

\section{INDEX}

Corollary III.11 allows us to apply all known results on 1D QCA to MPU's. In particular, the index theorem [1, which associates a rational number to each QCA with the property that two automata have the same index iff there is a continuous path of QCA connecting them. That is, the index characterizes the different possible phases of QCA.

In this section, we will show how the index theorem can be easily obtained in the framework of MPU, by using the results obtained in the previous section. Our approach has the additional benefit of providing a definition of the index which is very easy to compute. In the Appendix we will show how our definition coincides with the one given in Ref. 11 for QCA.

Let start defining the index of a tensor $\mathcal{U}$ in $\mathrm{CF}$ generating MPUs:

Definition IV.1. Take any $k$ so that the blocked tensor $\mathcal{U}_{k}$ is simple. Define $r$ and $\ell$ for $\mathcal{U}_{k}$ as in Definition III.6. The index of $\mathcal{U}$ is defined as ind $=\frac{1}{2}\left(\log _{2}(r)-\log _{2}(\ell)\right)$.

As compared to [1, we have included the logarithm in this definition since then the index has a more information-theoretical interpretation: It is the net flow of information to the right. Note that since $r \ell=d^{2}$, ind $=\log _{2}(r / d)=-\log _{2}(\ell / d)$.

The first thing to notice is that

Proposition IV.2. The index is well defined, that is, it does not depend on $k$.

Proof. We denote by $k_{0}$ the smallest $k$ so that $\mathcal{U}_{k}$ is simple and take $k>k_{0}$. Clearly, $\mathcal{U}_{k}$ is the result of blocking $\mathcal{U}_{k_{0}}$ with $\mathcal{U}_{k-k_{0}}$. In order to determine the index for such particular $k$, we combine indices to build a matrix and compute the rank as explained in the previous section. Graphically, for the first decomposition we have

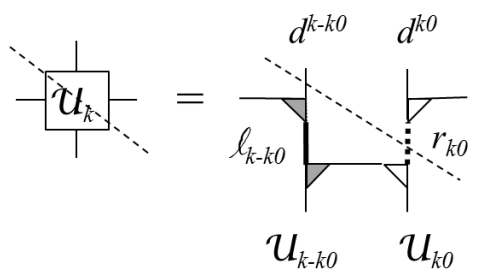

and similarly for the other one. According to this figure, the right rank, $r_{k}$ is upper bounded by $d^{k-k_{0}} r_{k_{0}}$. If we build the matrix the other way round, $\ell_{k}$ is upper bounded by $d^{k-k_{0}} \ell_{k_{0}}$. Applying Theorem III.8. since $\mathcal{U}_{k}$ is simple, we have that $r_{k} \ell_{k}=d^{k}$, and thus $r_{k}=d^{k-k_{0}} r_{k_{0}}$ and $\ell_{k}=d^{k-k_{0}} \ell_{k_{0}}$, so that the index is $\frac{1}{2}\left(\log \left(r_{k}\right)-\log \left(l_{k}\right)\right)=\frac{1}{2}\left(\log \left(r_{k_{0}}\right)-\log \left(\ell_{k_{0}}\right)\right)$.

Before stating and proving the Index Theorem for MPU, we need the following definition of equivalent tensors. Let us consider two tensors, $\mathcal{U}$ and $\mathcal{V}$, of physical dimensions $d_{a, b}$, respectively, generating MPU's, and let us denote by $p_{a, b}$ two coprimes such that $p_{a} d_{a}=p_{b} d_{b}$. We also denote by $\mathbb{1}_{x}$ the identity operator acting on a Hilbert space of dimension $x$, and by $\mathcal{U}^{(x)}=\mathcal{U} \otimes \mathbb{1}_{x}$, i.e. the tensor generating the MPV $U^{(N)} \otimes \mathbb{1}_{x}^{\otimes N}$.

Definition IV.3. Two tensors $\mathcal{U}$ and $\mathcal{V}$ in $C F$ are strictly equivalent if $d_{a}=d_{b}$ and there exist a continuous path $\mathcal{W}(p)$ of tensors, not necessarily in $C F$, with $p \in[0,1]$ such that $\mathcal{W}(0)=\mathcal{U}$ and $\mathcal{W}(1)=\mathcal{V}$.

Definition IV.4. Tensors $\mathcal{U}$ and $\mathcal{V}$ are equivalent if there exists some $k \in \mathbb{N}$ and $p_{a}, p_{b}$ such that $\mathcal{U}_{k}^{\left(p_{a}\right)}$ and $\mathcal{V}_{k}^{\left(p_{b}\right)}$ are strictly equivalent.

This last definition means that by attaching an ancilla of dimension $p_{a, b}$ and blocking, respectively, they can be locally transformed into each other.

Rather than saying that two MPUs $\mathcal{U}$ and $\mathcal{V}$ are equivalent or strictly equivalent, we will sometimes also say that they are in the same phase (or class) with regard to equivalence or strict equivalence; we will omit the notion of equivalence and just say that $\mathcal{U}$ and $\mathcal{V}$ are in the same phase when it is clear from the context.

It is important to make clear that we do not impose that the tensors in the path are in $\mathrm{CF}$ to allow the most general interpolation path, along which e.g. the bond dimension in CF might change, such as for the examples in Eq. 63). Since, however, the index is defined using the corresponding $\mathrm{CF}$ tensor, we will need the following result.

Proposition IV.5. Given a continuous map of tensors $[0,1] \ni x \mapsto \mathcal{W}(x)$, not necessarily in $C F$, generating $M P U$, consider the $C F$ and the ranks $r(x), \ell(x)$ of the associated simple tensors. Then both $r(x), \ell(x)$ are constant.

Proof. Let us consider any tensor $\mathcal{W}$, not necessarily in $\mathrm{CF}$, generating MPU. By blocking $D^{4}$ sites we can assume that its $\mathrm{CF}$ is simple and that its transfer operator is a rank-one map with positive semidefinite left and right fixed points which we call $L$ and $R$, respectively. That is, as a completely positive map, the transfer operator is $X \mapsto \operatorname{tr}(L X) R$, where $\operatorname{tr}(L R)=1$. This implies that

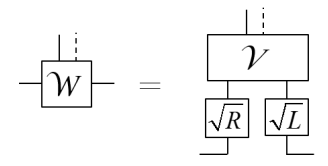


for an isometry $V$ and hence, if we call $P$ and $Q$ the projectors onto the support of $L$ and $R$ respectively, we have that $\mathcal{W} P=\mathcal{W}=Q \mathcal{W}$.

The first thing we will show is how we can use $P$ and $Q$ to obtain the the $\mathrm{CF}$ of $\mathcal{W}$. For that we invoke Jordan's Lemma, which ensures a decomposition of the space $\mathbb{C}^{D}=\left(\bigoplus_{i} \mathbb{C}^{2}\right) \oplus \mathbb{C}^{k}$ such that in that basis, $P=\bigoplus_{i}|0\rangle\left\langle\left. 0\right|_{i} \oplus R, P=\bigoplus_{i} \mid v_{i}\right\rangle\left\langle v_{i}\right| \oplus S$, where $R$ and $S$ are commuting projectors on $\mathbb{C}^{k}$. Let us define the projector $\tilde{P}:=\bigoplus_{i}|0\rangle\left\langle\left. 0\right|_{i} \oplus R S\right.$. We have the following properties:

(i) $P \tilde{P}=\tilde{P}$.

(ii) $P Q=\tilde{P} Q$.

(iii) $Q P=Q \tilde{P}$.

(iv) There exists an invertible $Y$ so that $\tilde{P} Q Y=\tilde{P}$.

We claim that $\tilde{\mathcal{W}}:=\tilde{P} \mathcal{W} \tilde{P}$ is the $\mathrm{CF}$ of $\mathcal{W}$, when restricted to the range of $\tilde{P}$. To see this, it is enough to show that both $\tilde{\mathcal{W}}$ and $\mathcal{W}$ define the same MPU for all $N$ and that the left and right fixed points of the transfer operator of $\tilde{\mathcal{W}}$ are full rank. The latter is obvious since the transfer operator of $\tilde{\mathcal{W}}$ is trivially $X \mapsto \operatorname{tr}(\tilde{P} L \tilde{P} X) \tilde{P} R \tilde{P}$, which implies that its left and right fixed points are, respectively, $\tilde{P} L \tilde{P}$ and $\tilde{P} R \tilde{P}$, which are both full rank in the range of $\tilde{P}$. The former is obvious from

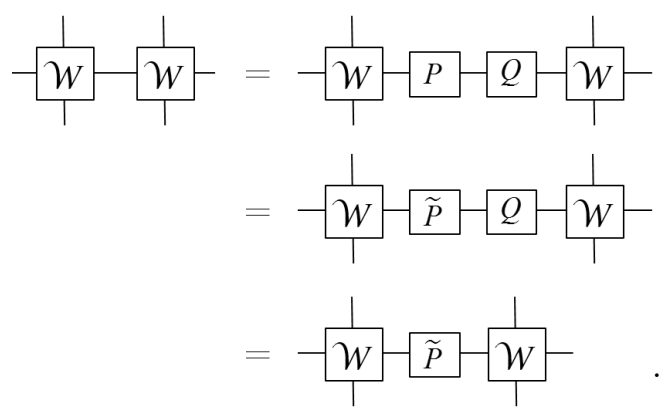

The next step is to define the tensor $\hat{\mathcal{W}}=L \mathcal{W} R$. We will prove that the rank along the cut

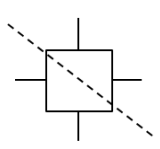

for $\hat{\mathcal{W}}$ and $\tilde{\mathcal{W}}$ is exactly the same (and similarly for the other cut). To this end, we take invertible matrices $X, Y, Z$ so that $X L=P, R Z=Q$ and $P Q Y=\tilde{P}$ (see (ii) and (iv) above). Then

$$
X \hat{\mathcal{W}} Z Y=P \mathcal{W} Q Y=P Q \mathcal{W} P Q Y=\tilde{P} \mathcal{W} \tilde{P}=\tilde{\mathcal{W}}
$$

which proves the claim. Moreover, since the CFII of $\tilde{\mathcal{W}}$ (restricted to the range of $\tilde{P}$ ) is constructed as $(\tilde{P} L \tilde{P})^{1 / 2} \tilde{W}(\tilde{P} L \tilde{P})^{-1 / 2}$, and $\tilde{P} L \tilde{P}$ is full rank of the range of $\tilde{P}$, the same holds for the corresponding ranks in CFII used to define the index, i.e., $r(x)$ and $\ell(x)$ are given by the corresponding ranks of $\hat{\mathcal{W}}(x)$.

Finally, given the continuous path $\mathcal{W}(x)$, it is clear that $\hat{\mathcal{W}}(x)$ is also continuous in $[0,1]$ since

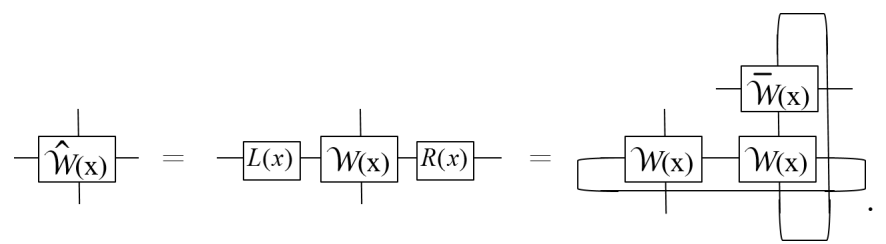

Since the rank of a continuous path of matrices is lower semicontinuous, both $r(x)$ and $\ell(x)$ are lower semicontinuous functions on $[0,1]$. Moreover, $r(x)=\frac{d^{2}}{\ell(x)}$, which implies that both $r(x)$ and $\ell(x)$ are also upper semicontinuous and hence continuous. Since they take integer values, they must be constant.

We can now state and prove the main result of this Section.

Theorem IV.6 (Index Theorem).

(i) The index does not change by blocking.

(ii) The index is additive by tensoring and composition.

(iii) The index is robust, i.e. by changing continuously $\mathcal{U}$ one cannot change it.

(iv) Two tensors have the same index iff they are equivalent.

Proof. (i) has been proven in Proposition IV.2.

(ii) The case of tensoring is trivial. To prove it for the concatenation of two MPU, we consider $\mathcal{U}$ and $\mathcal{U}^{\prime}$, with indices $\operatorname{ind}_{\mathcal{U}}$ and ind $_{\mathcal{U}^{\prime}}$, respectively. We define

$$
\frac{1}{-\frac{1}{1}}=\frac{-\frac{1}{u^{\prime}}-}{-\frac{1}{-}}
$$

where on the left we have double auxiliary indices, and denote by $\operatorname{ind}_{\mathcal{W}}$ its index. For that, we block $k$ times until $\mathcal{U}, \mathcal{U}^{\prime}$ and $\mathcal{W}_{k}$ are simple. We denote by $r_{k}, \ell_{k}$ and $r_{k}^{\prime}, \ell_{k}^{\prime}$ the corresponding ranks of $\mathcal{U}_{k}$ and $\mathcal{U}_{k}^{\prime}$. We now express $\mathcal{U}_{2 k}$ and $\mathcal{U}_{2 k}^{\prime}$ in SF and use them to build $\mathcal{W}_{4 k}$. We consider the matrix by joining indices in order to compute its right and left ranks, $r^{\prime \prime}$ and $\ell^{\prime \prime}$,

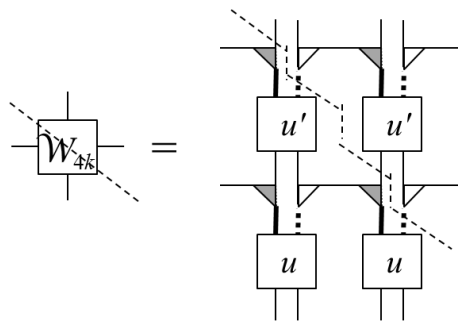


We have $r^{\prime \prime} \leq d_{k}^{2} r_{k} r_{k}^{\prime}$ and, similarly, $\ell^{\prime \prime} \leq d_{k}^{2} \ell_{k} \ell_{k}^{\prime}$. Since $\mathcal{W}_{4 k}$ is simple, $r^{\prime \prime} \ell^{\prime \prime}=d_{k}^{8}$ we conclude that $\operatorname{ind}_{\mathcal{W}}=$ $\frac{1}{2}\left(\log \left(r^{\prime \prime}\right)-\log \left(\ell^{\prime \prime}\right)\right)=\operatorname{ind}_{\mathcal{U}}+\operatorname{ind}_{\mathcal{U}^{\prime}}$.

(iii) has been proven in Proposition IV.5

(iv) Let us consider two tensors $\mathcal{U}$ and $\mathcal{V}$ and by ind $\mathcal{U}$ and ind $\mathcal{v}$ their indices.

(only if): Let us assume that $\operatorname{ind}_{\mathcal{U}} \neq \operatorname{ind}_{\mathcal{V}}$. Using (i) and (ii), and the fact that the identity operator has index equal to zero we have that the indices of $\mathcal{U}_{k}^{\left(p_{a}\right)}$ and $\mathcal{V}_{k}^{\left(p_{b}\right)}$ coincide with those of $\mathcal{U}$ and $\mathcal{V}$, and thus are different for any $k$. Thus, according to (iii), they cannot be continuously deformed into each other, so that they are not equivalent.

(if): if $\operatorname{ind}_{\mathcal{U}}=\operatorname{ind}_{\mathcal{V}}$, then we can find some $k$ such that both $\mathcal{U}_{k}^{\left(p_{a}\right)}$ and $\mathcal{V}_{k}^{\left(p_{b}\right)}$ are simple and have the same physical dimension. Then, the corresponding $u$ and $v$ defined in 290 have the same input and output dimensions, so that they can be smoothly connected to each other, and thus so can $\mathcal{U}_{k}^{\left(p_{a}\right)}$ and $\mathcal{V}_{k}^{\left(p_{b}\right)}$. Hence they are equivalent.

Let us now show that any continuous path corresponds to a continuous path in SF.

Corollary IV.7. For every continuous path of tensors $\mathcal{U}(p)$ - not necessarily in $C F$ - there exists $k_{0} \leq D^{4}$ and a continuous path $u(p)$ and $v(p)$ such that the $M P U$ $U^{\left(2 k_{0} N\right)}(p)$ generated by $\mathcal{U}(p)$ has a SF given by $u(p)$ and $v(p)$.

Proof. Let $\hat{\mathcal{U}}(p)$ be the CFII of $\mathcal{U}(p)$. Choose $k_{0} \leq D^{4}$ such that for all $p, \hat{\mathcal{U}}_{2 k_{0}}(p)$ is in SF, with unitaries $u(p)$ and $v(p)$. Note that the Index Theorem implies that $u(p): \mathbb{C}^{d} \otimes \mathbb{C}^{d} \rightarrow \mathbb{C}^{\ell} \otimes \mathbb{C}^{r}$ and $v(p): \mathbb{C}^{r} \otimes \mathbb{C}^{\ell} \rightarrow \mathbb{C}^{d} \otimes \mathbb{C}^{d}$ map between tensor product spaces of fixed dimensions, independent of $p$. Consider $U(p):=U^{\left(2 k_{0}\right)}(p)$, the MPU generated by $\mathcal{U}(p)$ [and equivalently $\hat{\mathcal{U}}(p)$ ] on $2 k_{0}$ sites. Since $\mathcal{U}(p)$ is continuous, so is $U(p)$. Thus, for any $x \in$ $[0,1]$ and $\varepsilon>0$ there exists a $\delta>0$ such that for all $|x-y|<\delta$,

$$
\left\|U(x) U(y)^{\dagger}-\mathbb{1}\right\|_{1} \leq \varepsilon .
$$

Substituting $U(p)=v_{23}(p) v_{41}(p) u_{12}(p) u_{34}(p)$, where the subscripts denote the $k_{0}$-blocked sites, and using unitary invariance of the trace norm, this yields

$$
\begin{aligned}
\| u_{12}(x) u_{12}^{\dagger}(y) \otimes & u_{34}(x) u_{34}^{\dagger}(y)- \\
& v_{23}^{\dagger}(x) v_{23}(y) \otimes v_{41}^{\dagger}(x) v_{41}(y) \|_{1} \leq \varepsilon .
\end{aligned}
$$

Now pick $|\alpha\rangle,|\beta\rangle,|\gamma\rangle,|\delta\rangle$ s.th. $C:=\left\langle\alpha,\left.\beta\right|_{34} u_{34}(x) \times\right.$ $u_{34}^{\dagger}(y)|\gamma, \delta\rangle_{34}$ satisfies $|C| \geq 1 / \sqrt{d}$, where $d$ is the dimension of the blocked site - e.g., by choosing $|\alpha, \beta\rangle$ the leading Schmidt vector of $u_{34}(x) u_{34}^{\dagger}(y)|\gamma, \delta\rangle_{34}$. Since $\left\|V X W^{\dagger}\right\|_{1} \leq\|X\|_{1}$ for isometries $V$ and $W$, we obtain with $V=\mathbb{1}_{12} \otimes\left\langle\alpha,\left.\beta\right|_{34} \text { and } W^{\dagger}=\mathbb{1}_{12} \otimes \mid \gamma, \delta\right\rangle_{34}$ from Eq. $40\left\|C u_{12}(x) u_{12}^{\dagger}(y)-\tilde{r}_{1}(x, y) \otimes \tilde{s}_{2}(x, y)\right\|_{1} \leq \varepsilon$ [with $\tilde{r}_{1}(x, y)=\left\langle\beta\left|{ }_{4} v_{14}^{\dagger}(x) v_{14}(y)\right| \delta\right\rangle_{4}$, and similarly for $\left.\tilde{s}_{2}(x, y)\right]$, and thus

$$
\left\|u_{12}(x)-\left(r_{1}(x, y) \otimes s_{2}(x, y)\right) u_{12}(y)\right\|_{1} \leq \sqrt{d} \varepsilon .
$$

We choose the relative norms of $r_{1}(x, y)$ and $s_{2}(x, y)$ such that $\left\|r_{1}(x, y)\right\|_{\infty}=1$.

While $r_{1}(x, y)$ and $s_{2}(x, y)$ are generally not unitary, we can replace them by the unitaries $r_{1}^{\prime}(x, y)$ and $s_{2}^{\prime}(x, y)$ obtained by setting their singular values to 1 , with a continuity bound

$\left\|u_{12}(x)-\left(r_{1}(x, y) \otimes s_{2}(x, y)\right) u_{12}(y)\right\|_{1} \leq\left(4 d^{2}+1\right) \sqrt{d} \varepsilon$.

This is easily shown from $\left\|u_{12}(x) u_{12}(y)^{\dagger}-r_{1} \otimes s_{2}\right\|_{1} \leq$ $\sqrt{d} \varepsilon$, which (using $\left\|r_{1}\right\|_{\infty}=1$ ) can be used to bound their singular values, yielding $\left\|r_{1} \otimes s_{1}-r_{1}^{\prime} \otimes s_{1}^{\prime}\right\|_{1} \leq 4 d^{2} \sqrt{d} \varepsilon$.

We thus see that $u_{12}(x)$, modulo local unitaries, is continuous in $x$. Using a classical result of Montgomery and Yang 23] (see Ref. 24] for a detailed explanation), it follows that there exists a local unitary gauge $\hat{r}(x) \otimes \hat{s}(x)$ such that $\hat{u}(x)=(\hat{r}(x) \otimes \hat{s}(x)) u(x)$ is continuous. It is then trivial to see that the corresponding $\hat{v}(p)$ is continuous as well, by using the continuity of $U^{\left(k_{0}\right)}(p)=\hat{u}(p) \mathbb{S} \hat{v}(p) \mathbb{S}$, where $\mathbb{S}$ swaps the indices of $\hat{v}(p)$.

\section{SYMMETRIES: LOCAL CHARACTERIZATION}

We will now turn our interest towards symmetries, and study the effect they have on the classification of MPUs, i.e., what happens when we impose some symmetry $U^{(N)}=\mathcal{S}\left[U^{(N)}\right]$ on the allowed MPUs. To this end, we will use the standard form for MPUs (Definition III.9) together with the local characterization of different MPU representations of the same unitary - namely $\mathcal{U}$ and $\mathcal{S}[\mathcal{U}]$ - established in Theorem $\amalg I I .10$.

We will consider the following three symmetries:

1. Conjugation $U^{(N)}=\overline{U^{(N)}}$

2. Time reversal $U^{(N)}=U^{(N)^{\dagger}}$

3. Transposition $U^{(N)}=U^{(N)^{T}}$

The ideas used can however be adapted, for instance to combine the above symmetries with local symmetry actions, such as in the case of topological insulators (where additionally the spin is flipped), but also to systems with other locally characterized symmetries.

In this section, we provide local characterizations of the three aforementioned symmetries based on Theorem III.10. We then apply these local characterizations in Sec. VI to classify the equivalence classes of MPUs under symmetries. Finally, we discuss a range of examples in Sec. VII.

The general procedure to analyze symmetries in MPU is analogous to that used for MPS 25]. Let us assume 
that for all $N$, the MPU generated by a tensor $\mathcal{U}$ is invariant under some symmetry, that is, $\tilde{U}^{(N)}=\mathcal{S}\left[U^{(N)}\right]=$ $U^{(N)}$. If we denote by $\tilde{\mathcal{U}}$ the tensor generating $\tilde{U}^{(N)}$, we have that both $\mathcal{U}$ and $\tilde{\mathcal{U}}$ generate the same family of MPU. Thus, if we block until we obtain the SF in both of them, then the resulting tensors have to be related by virtue of Theorem III.10. More explicitly, we will consider a tensor of the form (33), and define $u$ and $v$ as in (29). Thus, $u$ and $v$ fully characterize the MPU, and we just have to find the restrictions imposed by the symmetry. We will say that $u, v$ define the SF and that they generate the MPU.

\section{A. Conjugation}

We will consider MPU that are invariant under complex conjugation, that is, $\forall N: U^{(N)}=\overline{U^{(N)}}$. We will consider the SF defined by $u$ and $v$. This implies that $U^{(N)}$ and $\overline{U^{(N)}}$ must have the same index, since their corresponding dimensions $r$ and $\ell$ are equal.

Proposition V.1. The MPU generated by a tensor in SF are invariant under conjugation iff there exist unitaries $x, y$ fulfilling either $x=x^{T}, y=y^{T}$, or $x=$ $-x^{T}, y=-y^{T}$, such that

$$
\begin{aligned}
& \bar{u}=(x \otimes y) u, \\
& \bar{v}=v\left(y^{\dagger} \otimes x^{\dagger}\right),
\end{aligned}
$$

Proof. (if) It is immediate.

(only if) Let us assume that $U^{(N)}=\overline{U^{(N)}} \forall N$, where the conjugation is taken in the original basis where the MPU is defined. Using Theorem III.10, we obtain (41a) and 41b for some unitaries $x, y$. Using 41a twice, we obtain $\bar{x} x \otimes \bar{y} y=\mathbb{1}$, which is equivalent to $x=e^{i \phi} x^{T}$ and $y=e^{-i \phi} y^{T}$. This implies $\bar{x}=e^{-i \phi} x^{\dagger}$ and thus $\mathbb{1}=x x^{\dagger}=e^{2 i \phi} x^{T} \bar{x}=e^{2 i \phi} \mathbb{1}$, such that $e^{i \phi}= \pm 1$.

Let us now have a closer look at the structure of symmetric and skew-symmetric unitary matrices.

Lemma V.2. If a unitary matrix $x$ fulfills $x=x^{T}$ or $x=-x^{T}$, then there exist a symmetric unitary matrix $S, S=S^{T}$, and a $\tilde{\Lambda}$ such that $x=S^{T} \tilde{\Lambda} S$. Moreover, $\tilde{\Lambda}$ is real and symmetric (for $x=x^{T}$ ) or skew-symmetric (for $\left.x=-x^{T}\right)$, and $\operatorname{det}(\tilde{\Lambda})=1$.

Proof. Let us consider first the case $x=x^{T}$. Since $x$ is unitary, it has a spectral decomposition $x=\sum_{E} e^{-i E} P_{E}$ with $E \in[0,2 \pi)$, where the $\left\{P_{E}\right\}_{E}$ form a complete set of mutually orthogonal orthogonal projectors, and $P_{E}=$ $P_{E}^{T}$. Defining

$$
S=\sum_{E} e^{-i E / 2} P_{E}
$$

and $\tilde{\Lambda}=\mathbb{1}$, we have indeed that $S$ is unitary, $S=S^{T}$, and $x=S^{T} \tilde{\Lambda} S$.
Let us now consider $x=-x^{T}$. Since $x$ is unitary, we can write

$$
x=\sum_{E} e^{-i E}\left(P_{E}-P_{E}^{T}\right)
$$

where $E \in[0, \pi)$, and the $\left\{P_{E}, P_{E}^{T}\right\}_{E}$ form a complete set of mutually orthogonal orthogonal projectors. Defining

$$
S=e^{-i \pi / 4} \sum_{E} e^{-i E / 2}\left(P_{E}+P_{E}^{T}\right),
$$

and

$$
\tilde{\Lambda}=i \sum_{E}\left(P_{E}-P_{E}^{T}\right)
$$

we have that $S$ is unitary, $S=S^{T}$, and $x=S^{T} \tilde{\Lambda} S$. Moreover, $\overline{\tilde{\Lambda}}=-i \sum\left(\bar{P}_{E}-P_{E}^{\dagger}\right)=-i \sum\left(P_{E}^{T}-P_{E}\right)=\tilde{\Lambda}$, i.e., $\tilde{\Lambda}$ is real, and $\tilde{\Lambda}^{T}=-\tilde{\Lambda}$. Finally, since the eigenvalues of $\tilde{\Lambda}$ come in pairs $\pm i, \operatorname{det}(\tilde{\Lambda})=1$.

We now show that there always exist a gauge such that $u$ and $v$ can be choosen with special properties. This will be the basis of the classification of phases that we will carry out in the next section.

Proposition V.3. If the MPU generated by a tensor in $S F$ are invariant under complex conjugation, then one can choose the SF with unitaries $u^{\prime}$ and $v^{\prime}$ such that:

- Case I: If $x=x^{T}$ and $y=y^{T}$ in Prop. V.1 then

$$
\overline{u^{\prime}}=u^{\prime}, \quad \overline{v^{\prime}}=v^{\prime},
$$

i.e. they are orthogonal. The choice of $u^{\prime}$ and $v^{\prime}$ is unique up to local orthogonal transformations; that is, if $u^{\prime \prime}$ and $v^{\prime \prime}$ are orthogonal and generate the same $M P U$, then there exist $O_{r, \ell}$ orthogonal, such that $u^{\prime \prime}=\left(O_{\ell} \otimes O_{r}\right) u^{\prime}$ and $v^{\prime \prime}=v^{\prime}\left(O_{r}^{T} \otimes O_{\ell}^{T}\right)$.

- Case II: If $x=-x^{T}$ and $y=-y^{T}$ in Prop. V.1. then

$$
\overline{u^{\prime}}=\left(\Sigma_{\ell} \otimes \Sigma_{r}\right) u^{\prime}, \quad \overline{v^{\prime}}=v^{\prime}\left(\Sigma_{r} \otimes \Sigma_{\ell}\right),
$$

where $\Sigma_{x}=\mathbb{1}_{x / 2} \otimes Y, x=r, \ell$, and $Y=\left(\begin{array}{cc}0 & 1 \\ -1 & 0\end{array}\right)$. This choice is unique up to local symplectic transformations; that is, if $u^{\prime \prime}$ and $v^{\prime \prime}$ fulfill (43) and generate the same $M P U$, then there exist $S_{r, \ell}$ fulfilling $S_{x}^{T} \Sigma_{x} S_{x}=\Sigma_{x}$ for $x=r$, $\ell$ such that $u^{\prime \prime}=$ $\left(S_{\ell} \otimes S_{r}\right) u^{\prime}$ and $v^{\prime \prime}=v^{\prime}\left(S_{r}^{T} \otimes S_{\ell}^{T}\right)$. Note that this case can only occur if $\ell$ and $r$ are even.

Proof. First, it holds that every unitary with $x= \pm x^{T}$ can be written as $x=U^{T} \Lambda U$ with $U$ unitary, where for $x=x^{T}, \Lambda=\mathbb{1}$, while for $x=-x^{T}, \Lambda=\Sigma$. This follows directly from Lemma $\mathrm{V} .2$ and its proof: For $x=x^{T}$, this is immediate with $U=S$. For $x=-x^{T}$, we use that $\tilde{\Lambda}$ is real and skew-symmetric and can thus be block-diagonalized with an orthogonal transformation $O$, $O^{T} \Sigma O=\tilde{\Lambda}$, and then choose $U=O S$. (Alternatively, 
this can be derived from the Autonne-Takagi factorization or Youla's decomposition of (skew-)symmetric matrices under unitary congruence, respectively, using the unitarity of $x$.)

Now consider $u$ and $v$ describing an MPU satisfying (41), and write $x=U^{T} \Lambda_{\ell} U$ and $y=V^{T} \Lambda_{r} V$ with $U$, $V$ unitary. Define $u^{\prime}:=(U \otimes V) u$ and $v^{\prime}:=v\left(V^{\dagger} \otimes\right.$ $\left.U^{\dagger}\right)$. Clearly, $u^{\prime}$ and $v^{\prime}$ describe the same MPU, and it is straightforward to check that $\bar{u}^{\prime}=\left(\Lambda_{\ell} \otimes \Lambda_{r}\right) u^{\prime}$ and $\bar{v}^{\prime}=v^{\prime}\left(\Lambda_{r}^{T} \otimes \Lambda_{\ell}^{T}\right)=v^{\prime}\left(\Lambda_{r} \otimes \Lambda_{\ell}\right)$, which proves the first statement for both cases.

Now, if $u^{\prime \prime}, v^{\prime \prime}$ generate the same MPU as $u^{\prime}, v^{\prime}$, according to Theorem III.10 they must be related by some unitaries, ie $u^{\prime \prime}=(a \otimes b) u^{\prime}$ and $v^{\prime \prime}=v^{\prime}\left(b^{\dagger} \otimes a^{\dagger}\right)$. If $u^{\prime \prime}$ and $v^{\prime \prime}$ are orthogonal, then so is $a \otimes b$, and thus $\bar{a}=e^{i \phi} a, \bar{b}=e^{-i \phi} b$. Choosing $O_{\ell}=e^{-i \phi / 2} a$ and $O_{r}=e^{i \phi / 2} b$ finishes the proof for Case I. If $u^{\prime \prime}$ and $v^{\prime \prime}$ fulfill 43, then it is straightforward to show that $(a \otimes b)\left(\Sigma_{\ell} \otimes \Sigma_{r}\right)(a \otimes b)=\left(\Sigma_{\ell} \otimes \Sigma_{r}\right)$, and thus $a$ and $b$ can be chosen symplectic by an appropriate phase choice, as announced in Case II.

The second case in this proposition may look more intricate that the first one. However, there exists a simple characterization for the unitaries $u^{\prime}, v^{\prime}$.

Corollary V.4. In Case II of Proposition V.3 we can write

$$
\begin{aligned}
& u^{\prime}=\left(\mathbb{1}_{r \ell / 4} \otimes R\right) \tilde{u} \\
& v^{\prime}=\tilde{v}\left(\mathbb{1}_{r \ell / 4} \otimes R\right)
\end{aligned}
$$

with $R=\frac{1-i}{2}\left[\mathbb{1}_{4}+i(Y \otimes Y)\right]$ unitary, and $\tilde{u}$ and $\tilde{v}$ orthogonal, where the ordering of the tensor product is such that the positions of the $Y$ coincide with those in $\Sigma_{x}$ in (43).

Proof. This can be obtained by noticing that $R^{T}(Y \otimes$ $Y) R=\mathbb{1}_{4}$.

We emphasize, however, that the resulting $\tilde{u}$ and $\tilde{v}$ do not generate the same MPU. Indeed, we will see that the MPU generated by $\tilde{u}, \tilde{v}$ and by $u^{\prime}, v^{\prime}$ are in fact in different phases under conjugation.

\section{B. Time-reversal}

We consider now the case where $U^{(N)}=U^{(N) \dagger}$. The first thing to notice is that this is impossible unless the index of the generating tensor vanishes. Indeed, if it is non-zero, the tensors generating $U^{(N)}$ and $U^{(N) \dagger}$ cannot be equivalent, since their indices are opposite, a result that directly follows from Theorem IV.6 and the fact that $U^{(N)} U^{(N) \dagger}=\mathbb{1}$ has index equal to zero.

In the following we will consider the SF so that there are two spins per site, $H_{d}=H_{d_{0}} \otimes H_{d_{0}}$, i.e. in site $n$ we have spins $2 n-1$ and spin $2 n$. We will use a subscript to indicate the spin when required.
Proposition V.5. The MPU generated by a tensor in $S F$ are invariant under chirality, $U^{(N)}=U^{(N) \dagger}$ for $N$ even, iff there exists a unitary $x$ such that

$$
\left(\mathbb{1}_{1} \otimes v_{23} \otimes \mathbb{1}_{4}\right)\left(u_{12} \otimes u_{34}\right)= \pm\left(x_{12} \otimes x_{34}^{\dagger}\right)\left(\mathbb{1}_{1} \otimes v_{23}^{\dagger} \otimes \mathbb{1}_{4}\right)
$$

We will discuss the relevance of the \pm 1 phase for the classification of phases in Sec. VIB.

Proof. (if) For even $N$, we have

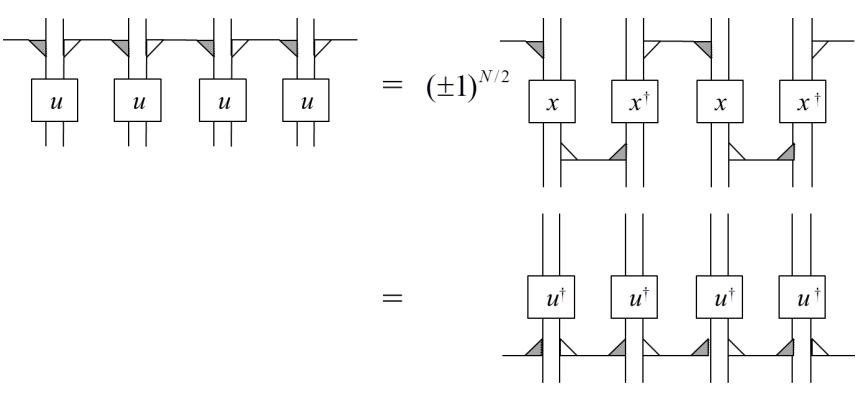

where we first used (45) and then its daggered version.

(only if) The tensor generating $U^{(N) \dagger}$ is not in SF, so that we cannot use the fundamental theorem. The way around this is to block two tensors, so that

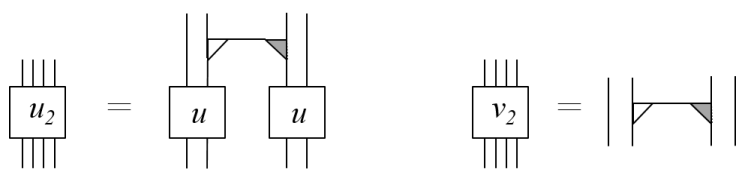

Using Theorem III.10 [Eq. (35a)] for the blocked MPU, we have $\left(\mathbb{1}_{1} \otimes v_{23} \otimes \mathbb{1}_{4}\right)\left(u_{12} \otimes u_{34}\right)=\left(x_{12} \otimes y_{34}\right)\left(\mathbb{1}_{1} \otimes\right.$ $\left.v_{23}^{\dagger} \otimes \mathbb{1}_{4}\right)$. Analogously, using Eq. $35 \mathrm{~b}$ we deduce that $\left(\mathbb{1}_{1} \otimes v_{23} \otimes \mathbb{1}_{4}\right)=\left(u_{12}^{\dagger} \otimes u_{34}^{\dagger}\right)\left(\mathbb{1}_{1} \otimes v_{23}^{\dagger} \otimes \mathbb{1}_{4}\right)\left(y_{12}^{\dagger} \otimes x_{34}^{\dagger}\right)$. Substituting the dagger of the second equation into the first one, we obtain $x_{12} y_{12} \otimes y_{34} x_{34}=\mathbb{1}$, and thus $y=$ $\pm x^{\dagger}$.

\section{Transposition}

Now we will assume that $U^{(N)}=U^{(N) T}$. Again, we can only have this symmetry if the index vanishes, since the indices of $U^{(N)}$ and $U^{(N)^{T}}$ are opposite - this can be seen by blocking as in Eq. 47) and using Definition IV.1 of the index in terms of $r$ and $\ell$.

Proposition V.6. The MPU generated by a tensor in $S F$ are invariant under transposition, $U^{(N)}=U^{(N) T}$ for $N$ even, iff there exists a unitary, $x$, such that

$$
\left(\mathbb{1}_{1} \otimes v_{23} \otimes \mathbb{1}_{4}\right)\left(u_{12} \otimes u_{34}\right)=e^{i \phi}\left(x_{12} \otimes x_{34}^{T}\right)\left(\mathbb{1}_{1} \otimes v_{23}^{T} \otimes \mathbb{1}_{4}\right)
$$

Proof. The proof is completely analogous to that of Proposition V.5 just that the phase cannot be fixed to \pm 1 . This can also be understood from the fact that in the derivation of the "if" direction, the phase cancels out, unlike in the proof of Prop. V.5 where it adds up. 
Note that the phase $e^{i \phi}$ in Eq. 48 bears no relevance for the classification of phases: It can be continuously changed to $\phi=0$ while keeping the symmetry under transposition by replacing $u \rightsquigarrow e^{-i \theta / 2} u$ and changing $\theta$ from 0 to $\phi$.

\section{Other symmetries}

We conclude this section by noting that other important symmetries involving any local unitary operator, $Q=Q^{\dagger}=Q^{T}$, can be reduced to the ones above. For instance:

$$
\begin{aligned}
{\left[Q^{\otimes N}\right] U^{(N) \dagger}\left[Q^{\otimes N}\right] } & =U^{(N)} \\
{\left[Q^{\otimes N}\right] U^{(N) T}\left[Q^{\otimes N}\right] } & =U^{(N)}
\end{aligned}
$$

can be reduced to the previous ones by defining $\tilde{\mathcal{U}}=$ $\mathcal{U} Q$, so that the MPU generated by those new tensors are invariant under time reversal and transposition. The most interesting case is the one where $Q=\mathbb{S}$, i.e. the swap operator

$$
\mathbb{S}(a \otimes b) \mathbb{S}=b \otimes a
$$

exchanging the two physical indices in the SF (33), as we will see in some examples.

We conclude this section by just mentioning that a similar approach to the one developed here, based on the fundamental theorem of MPUs can be applied to other symmetries, like reflection or global symmetries.

\section{SYMMETRIES: EQUIVALENCES}

In the following, we consider the equivalence of MPUs under symmetry constraints. For the case of conjugation, we will classify all possible phases. In the other cases, we will give necessary conditions for two MPUs to be in the same phase. In the next section we will illustrate these results with some examples.

In full analogy to the definition of strict equivalence (Def. IV.3) and equivalence (Def. IV.4) of MPU tensors, we will consider the following two notions of equivalence under symmetry:

Definition VI.1. Two tensors $\mathcal{U}$ and $\mathcal{V}$ are strictly equivalent under the symmetry $\mathcal{S}$ if $d_{a}=d_{b}$ and there exist a continuous path $\mathcal{W}(p), p \in[0,1]$, not necessarily in canonical form, such that $\mathcal{W}(0)=\mathcal{U}$ and $\mathcal{W}(1)=\mathcal{V}$, where the $M P U W(p)^{(N)}$ defined by $\mathcal{W}(p)$ is invariant under $\mathcal{S}, \mathcal{S}\left[W(p)^{(N)}\right]=S\left[W(p)^{(N)}\right]$.

Definition VI.2. Two tensors $\mathcal{U}$ and $\mathcal{V}$ are equivalent under the symmetry $\mathcal{S}$ if there exists some $k \in \mathbb{N}$ and $p_{a}, p_{b}$ such that $\mathcal{U}_{k}^{\left(p_{a}\right)}$ and $\mathcal{V}_{k}^{\left(p_{b}\right)}$ (the tensors obtained by blocking $k$ sites and adding ancillas of dimension $p_{a}$ and $p_{b}$, respectively) are strictly equivalent under the symme$\operatorname{try} \mathcal{S}$.
As we will see below, unlike the case without symmetries, these definitions give different results. The motivation of keeping both definitions is that the second involve that $N$ is a multiple of $k$, and thus may be restrictive when considering finite systems. Since we will assume SF for all the MPUs we consider here, we will characterize the equivalence under symmetries in terms of the unitaries $u, v$ that define them:

Lemma VI.3. Two MPU, $U_{1,2}^{(N)}$ described by simple tensors are strictly equivalent under the symmetry $\mathcal{S}$ iff there exist two continuous families of unitary matrices $\tilde{u}(p), \tilde{v}(p), p \in[0,1]$ such that $\tilde{u}(0)=\tilde{u}_{1}, \tilde{v}(0)=\tilde{v}_{1}$, generate $U_{1}^{(N)}$, and $\tilde{u}(1)=\tilde{u}_{2}$, and $\tilde{v}(1)=\tilde{v}_{2}$ generate $U_{2}^{(N)}$ and the MPU generated by $\tilde{u}(p), \tilde{v}(p)$ are invariant under $\mathcal{S}$.

Proof. The proof follows directly from Corollary IV.7.

\section{A. Equivalences under Conjugation}

We consider two simple tensors, $\mathcal{U}_{1,2}$ generating MPUs that are invariant under conjugation and are interested in determining when they are equivalent or strictly equivalent under that symmetry. In SF, they are generated by some $u_{1}, v_{1}$ and $u_{2}, v_{2}$, which according to Theorem III.10 are only fixed up to a unitary gauge $u_{i} \rightsquigarrow(x \otimes y) u_{i}$, $v_{i}=v_{i}\left(y^{\dagger} \otimes x^{\dagger}\right)$. According to Lemma VI.3, we thus want to know the precise conditions under which we can choose $u_{1}, v_{1}$ and $u_{2}, v_{2}$ such that they can be connected with a continuous path $u(p), v(p)$ which define MPUs with the same symmetry. We will consider two cases: (i) at least one of $r, \ell$ are odd; (ii) both $r$ and $\ell$ are even.

\section{At least one of $r, \ell$ are odd}

If at least one of $r, \ell$ is odd, only Case I in Proposition V.3 can occur.

Theorem VI.4. If either $r$ or $\ell$ is odd, $\mathcal{U}_{1,2}$ are strictly equivalent under conjugation symmetry iff

$$
\operatorname{det}\left(u_{1} v_{1}\right)=\operatorname{det}\left(u_{2} v_{2}\right) \text {. }
$$

Note that $\operatorname{det}\left((x \otimes y) u_{i} v_{i}\left(y^{\dagger} \otimes x^{\dagger}\right)\right)=\operatorname{det}(x \otimes y) \times$ $\operatorname{det}\left(u_{i} v_{i}\right) \operatorname{det}\left(y^{\dagger} \otimes x^{\dagger}\right)=\operatorname{det}\left(u_{i} v_{i}\right)$, i.e. $\operatorname{det}\left(u_{i} v_{i}\right)$ is gauge invariant (as required).

Proof. (only if) Starting from Proposition V.1 it immediately follows that $\operatorname{det}\left(u_{i} v_{i}\right)= \pm 1$. Since the sign of this determinant is a continuous function of $u$ and $v$, we conclude that if they are equivalent this determinant must be the same.

(if) We will explicitly construct the continuous path $u(p)$, $v(p)$. To this end, we first choose a suitable gauge for the SF $u_{i}$ and $v_{i}, i=1,2$ of the endpoints $\mathcal{U}_{i}$. Since $r$ 
and $\ell$ are not both even, we are in Case I of Proposition V.3, i.e., we can choose a gauge where $u_{i}$ and $v_{i}$ are orthogonal. Furthermore, if $\operatorname{det}\left(u_{1}\right)=-\operatorname{det}\left(u_{2}\right)$ [and thus $\left.\operatorname{det}\left(v_{1}\right)=-\operatorname{det}\left(v_{2}\right)\right]$, and w.l.o.g. $r$ odd, we can find an orthogonal $\ell \times \ell$ matrix, $O_{\ell}$, with $\operatorname{det}\left(O_{\ell}\right)=$ -1 , and change the gauge as $u_{2} \mapsto\left(O_{\ell} \otimes \mathbb{1}_{r}\right) u_{2}$ and $v_{2} \mapsto v_{2}\left(\mathbb{1}_{r} \otimes O_{\ell}^{T}\right)$, which multiplies their determinants by $\operatorname{det}\left(O_{\ell}\right)^{r}=-1$.

We thus see that we can always find a gauge with $u_{i}$, $v_{i}$ orthogonal and $\operatorname{det}\left(u_{1}\right)=\operatorname{det}\left(u_{2}\right), \operatorname{det}\left(v_{1}\right)=\operatorname{det}\left(v_{2}\right)$. Then, since the group of orthogonal transformations with a fixed determinant is simply connected, we can always find a smooth orthogonal interpolation $u(p), v(p)$ between them. But the fact that it is orthogonal, according to Proposition V.1, implies that it satisfies the symmetry, so that this finishes the proof.

Corollary VI.5. AllU are equivalent under conjugation symmetry.

Proof. Given $u, v$ generating MPU with conjugation symmetry, if we block once we will have that the new $u^{(2)}, v^{(2)}$ can be written as $u_{11^{\prime}, 22^{\prime}}^{(2)}=v_{1^{\prime} 2} u_{11^{\prime}} u_{22^{\prime}}$ and $v_{11^{\prime}, 22^{\prime}}^{(2)}=$ $v_{1^{\prime} 2}$, so that $\operatorname{det}\left(u^{(2)} v^{(2)}\right)=\operatorname{det}(u v)^{2 r \ell}=1$. By applying the previous proposition we arrive at the conclusion.

\section{2. $r$ and $\ell$ are both even}

Now, both Case I and Case II in Proposition V.3 can occur. Since we can continuously change the gauge for $u, v$ without affecting the symmetry, we will assume that $u, v$ fulfill (42) and 43) in Case I and II, respectively. With this choice,

$$
\operatorname{det}\left(u_{i}\right)= \pm 1, \quad \operatorname{det}\left(v_{i}\right)= \pm 1
$$

as follows directly from 42 and 43 . Moreover, $\operatorname{det}\left(u_{i}\right)$ and $\operatorname{det}\left(v_{i}\right)$ do not depend on the specific choice of $u_{i}$ and $v_{i}$, since the determinant of the remaining orthogonal or sympectic degree of freedom is $\operatorname{det}\left(O_{\ell} \otimes O_{r}\right)=\operatorname{det}\left(S_{\ell} \otimes\right.$ $\left.S_{r}\right)=+1$ (since $r$ and $\ell$ are even). We will henceforth use this choice of $u_{i}$ and $v_{i}$ for the SF of $\mathcal{U}_{i}$.

Theorem VI.6. If $r$ and $\ell$ are both even, two $M P U \mathcal{U}_{1,2}$, with $u_{i}$ and $v_{i}$ for their SF fulfilling (42) or (43), respectively, are strictly equivalent under conjugation symmetry iff

$$
\operatorname{det}\left(u_{1}\right)=\operatorname{det}\left(u_{2}\right), \quad \operatorname{det}\left(v_{1}\right)=\operatorname{det}\left(v_{2}\right)
$$

and moreover they correspond to the same case in Proposition $V .3$.

Proof. (only if) First, let us assume $\mathcal{U}_{1,2}$ correspond to different cases in Prop.V.3. Then, according to Prop.V.1 since $y \otimes x=v^{T} v=\mathbb{S} \bar{u} u^{\dagger} \mathbb{S}^{\dagger}$ (with $\mathbb{S}$ the swap), $x \otimes y$ is unitary and continuous in $u, v$, and thus, we can choose $x$ and $y$ unitary and continuous as well, so that they cannot change from symmetric to skew-symmetric.
Let us thus now consider the case where $\mathcal{U}_{1,2}$ correspond to the same case in Prop. V.3, and assume there is a path $u(p), v(p)$ that keeps the symmetry and that connects them continuously. Thus, $u(p)$ and $v(p)$ fulfill Proposition V.1 with some $x(p), y(p)$. Since we can write $x(p) \otimes y(p)=\bar{u}(p) u(p)^{\dagger}$, they can also be chosen continuous. Using Lemma V.2 we can write $x(p)=S_{x}(p)^{T} \tilde{\Lambda}_{x}(p) S_{x}(p)$ and $y(p)=S_{y}(p)^{T} \tilde{\Lambda}_{y}(p) S_{y}(p)$ with $S_{x, y}(p)=S_{x, y}(p)^{T}$ unitary. Since $\operatorname{det}\left[\tilde{\Lambda}_{x, y}(p)\right]=1$, we have that $\operatorname{det}\left[S_{x, y}(p)\right]^{2}$ is continuous as well. Performing the gauge transformation

$$
\begin{aligned}
& u^{\prime}(p)=\left[S_{x}(p) \otimes S_{y}(p)\right] u(p), \\
& v^{\prime}(p)=v(p)\left[S_{y}(p)^{\dagger} \otimes S_{x}(p)^{\dagger}\right]
\end{aligned}
$$

we have that $u^{\prime}, v^{\prime}$ generate the same MPU as $u, v$. Furthermore, $\operatorname{det}\left[u^{\prime}(p)\right]=\operatorname{det}\left[S_{x}(p)\right]^{r} \operatorname{det}\left[S_{y}(p)\right]^{\ell} \operatorname{det}[u(p)]$ is continuous in $p$ since both $r$ and $\ell$ are even, and $u(p)$ is continuous. But since $\overline{u^{\prime}(p)}=\left[\tilde{\Lambda}_{x}(p) \otimes \tilde{\Lambda}_{y}(p)\right] u^{\prime}(p)$, and $\operatorname{det}\left[\tilde{\Lambda}_{x, y}(p)\right]=1$, we have that $\operatorname{det}\left[u^{\prime}(p)\right]= \pm 1$. Thus, we must have $\operatorname{det}\left[u^{\prime}(0)\right]=\operatorname{det}\left[u^{\prime}(1)\right]$. Finally, following the proof of Prop. V.3 the gauge transformations $O_{x, y}(0), O_{x, y}(1)$ bringing $u^{\prime}(0)$ and $u^{\prime}(1)$ into the form $u_{1}$ and $u_{2}$ fulfilling 42 or 43, respectively, are orthogonal, and since $r$ and $\ell$ are even they do not change the sign of the determinant, such that $\operatorname{det}\left[u_{1}\right]=\operatorname{det}\left[u^{\prime}(0)\right]=$ $\operatorname{det}\left[u^{\prime}(1)\right]=\operatorname{det}\left[u_{2}\right]$. The result for $v_{1}$ and $v_{2}$ follows accordingly.

(if) Since both MPU are in the same case of Prop. V.3. we can proceed as in Theorem VI.4. In Case I, since $u_{1}$ and $u_{2}$ are orthogonal and have the same determinant, they can be continuously connected with $u(p)$ orthogonal. In Case II, we can always write $u_{i}, v_{i}$ in the form 44 , i.e. $u_{i}=\left(\mathbb{1}_{r \ell / 4} \otimes R\right) \tilde{u}_{i}$ with some orthogonal $\tilde{u}_{i}$ with $\operatorname{det}\left(\tilde{u}_{i}\right)=\operatorname{det}\left(\tilde{v}_{i}\right)$, and correspondingly for $v_{i}$, which can thus be connected by choosing a continuous path of orthogonal matrices $\tilde{u}(p)$ and $\tilde{v}(p)$.

Corollary VI.7. $\mathcal{U}_{1,2}$ are equivalent under conjugation symmetry iff they are in the same case according to Proposition $V .3$.

Proof. First, choose $u$ and $v$ such that they satisfy 42 or 43, respectively. By blocking once, we obtain new $u_{11^{\prime}, 22^{\prime}}^{(2)}=v_{1^{\prime} 2} u_{11^{\prime}} u_{22^{\prime}}$ and $v_{11^{\prime}, 22^{\prime}}^{(2)}=v_{1^{\prime} 2}$, so that $\operatorname{det}\left(u^{(2)}\right)=\left(\operatorname{det}(u)^{2} \operatorname{det}(v)\right)^{r \ell}=1$ and $\operatorname{det}\left(v^{(2)}\right)=$ $(\operatorname{det}(v))^{r \ell}=1$. Moreover, it is straightforward to check that $u^{(2)}$ and $v^{(2)}$ still satisfy 42 or 43) (in the latter case with $\Sigma_{\ell}^{(2)}=\Sigma_{\ell} \otimes \mathbb{1}_{r}$ and $\Sigma_{r}^{(2)}=\mathbb{1}_{\ell} \otimes \Sigma_{r}$ ), and thus by applying Thm. VI.6, we arrive at the conclusion.

\section{B. Necessary conditions under time reversal symmetry}

In the following, we will consider necessary conditions for equivalence under symmetries which can be derived 
from the local characterization of symmetries in Section V. Since the local characterization of transposition symmetry, Prop. V.6 leads to no non-trivial invariants (the phase in Eq. (48) can be changed smoothly while keeping the symmetry, cf. the comment after the proposition), the remaining case of interest is time reversal symmetry.

Let us start by recalling the local characterization of time reversal symmetry in Prop. V.5 for an MPU in SF, Eq. (45), which we here write for conciseness with subscripts denoting the sites each operator acts on:

$$
v_{23} u_{12} u_{34}=\sigma x_{12} x_{34}^{\dagger} v_{23}^{\dagger}
$$

with $\sigma= \pm 1$. Note that $\sigma$ can be extracted directly from $u$ and $v$ :

Lemma VI.8. For a time reversal invariant $M P U$ in $S F$ represented by $u$ and $v, \sigma$ in Eq. (52) is given by

$$
\sigma=\frac{1}{d^{2}} \operatorname{tr}\left[\mathbb{S}_{12,34} v_{23} u_{12} u_{34} v_{23}\right]
$$

where $\mathbb{S}_{12,34}$ swaps sites 12 with 34 .

Proof. This follows directly from $v_{23} u_{12} u_{34} v_{23}=$ $\sigma x_{12} x_{34}^{\dagger}$, together with the fact that $\operatorname{tr}[\mathbb{S}(a \otimes b)]=\operatorname{tr}[a b]$ and thus $\operatorname{tr}\left[\mathbb{S}_{12,34} x_{12} x_{34}^{\dagger}\right]=\operatorname{tr}\left[x x^{\dagger}\right]=d^{2}$.

Let us now show that the sign is gauge invariant and cannot be changed by continuous deformations.

Proposition VI.9. For an $M P U \mathcal{U}$ in $S F$ represented by $u$ and $v, \sigma= \pm 1$ given by Eq. (52) is well defined (i.e., independent of the gauge) and cannot be changed by continuous deformations of $\mathcal{U}$ which keep time reversal symmetry.

Proof. It is straightforward to check that the r.h.s. of (53) is invariant under gauge tranformations $u \rightsquigarrow(x \otimes y) u$, $v \rightsquigarrow v\left(y^{\dagger} \otimes x^{\dagger}\right)$. Also, (53) implies that $\sigma$ is continuous (and thus constant) under continuous changes of $u, v$ which keep time reversal symmetry.

This demonstrates that $\sigma$ is an invariant characterizing inequivalent classes of MPUs. In principle, there can be a different $\sigma$ for each level of blocking, which all form invariants; for instance, $u=i \mathbb{1}$ and $v=\mathbb{1}$ has $\sigma=-1$, while after blocking $k=2$ sites, we have $u^{(2)}=-\mathbb{1}$ and $v^{(2)}=\mathbb{1}$ which have $\sigma^{(2)}=+1$. However, as we now show, the phases $\sigma \equiv \sigma^{(1)}$ and $\sigma^{(2)}$ obtained in Eq. 52 after blocking 1 and 2 sites, respectively, completely determine the phase for all other blockings.

Proposition VI.10. Let $\mathcal{U}$ be a time reversal invariant $M P U \mathcal{U}$ in SF described by $u, v$, and let the blocked $M P U$ $\mathcal{U}_{k}$ be described by $u^{(k)}, v^{(k)}$. Denote by $\sigma^{(k)}$ the phase obtained from $u^{(k)}$ and $v^{(k)}$ in Eq. (52). Then,

$$
\sigma^{(k)}= \begin{cases}\sigma^{(1)} & \text { if } k \text { odd } \\ \sigma^{(2)} & \text { if } k \text { even }\end{cases}
$$

Proof. Because of Prop. VI.9, we can choose the representations $u^{(k)}, v^{(k)}$ at will. For now, we choose $u_{1234}^{(2)}=v_{23} u_{12} u_{34}$ and $v_{3456}^{(2)}=v_{45}$. Since blocking preserves the symmetry, we have that

$$
v_{45} u_{34} u_{56} v_{45}=\sigma^{(1)} x_{34} x_{56}^{\dagger}
$$

(where we use site labels $3,4,5,6$ ) and

$$
\begin{aligned}
\sigma^{(2)} X_{1234} X_{5678}^{\dagger} & =v_{3456}^{(2)} u_{1234}^{(2)} u_{5678}^{(2)} v_{3456}^{(2)} \\
& =v_{23} v_{45} v_{67} u_{12} u_{34} u_{56} u_{78} v_{45} \\
& \stackrel{54}{=} \sigma^{(1)} v_{23} v_{67} u_{12} u_{78} x_{34} x_{56}^{\dagger}
\end{aligned}
$$

From this, we infer (by equating the terms acting on sites 1234 with $\left.X_{1234}\right)$ that

$$
\zeta[(\mathbb{1} \otimes v \otimes \mathbb{1})(u \otimes x)]^{\dagger}=(\mathbb{1} \otimes v \otimes \mathbb{1})\left(x^{\dagger} \otimes u\right),
$$

where $\zeta=\sigma^{(2)} / \sigma^{(1)}=\sigma^{(1)} \sigma^{(2)}$. Now consider $k$ blocked sites, where we choose

$$
\begin{aligned}
& u^{\prime} \equiv u^{(k)}=\left(\mathbb{1} \otimes v^{\otimes k-1} \otimes \mathbb{1}\right)\left(u^{\otimes k}\right) \\
& v^{\prime} \equiv v^{(k)}=\mathbb{1}^{\otimes(k-1)} \otimes v \otimes \mathbb{1}^{\otimes(k-1)}
\end{aligned}
$$

Eq. (52) with $k$ blocked sites now reads

$$
X \otimes Y=\left(\mathbb{1}^{\otimes k} \otimes v^{\prime} \otimes \mathbb{1}^{\otimes k}\right)\left(u^{\prime} \otimes u^{\prime}\right)\left(\mathbb{1}^{\otimes k} \otimes v^{\prime} \otimes \mathbb{1}^{\otimes k}\right),
$$

where $\sigma^{(k)}$ can be obtained from $Y=\sigma^{(k)} X^{\dagger}$ (this relation must hold with some phase as blocking preserves the symmetry). By using (54) on the two middle sites, we find that

$$
\begin{aligned}
& X=\left(\mathbb{1} \otimes v^{\otimes(k-1)} \otimes \mathbb{1}\right)\left(u^{\otimes(k-1)} \otimes x\right), \\
& Y=\sigma^{(1)}\left(\mathbb{1} \otimes v^{\otimes(k-1)} \otimes \mathbb{1}\right)\left(x^{\dagger} \otimes u^{\otimes(k-1)}\right) .
\end{aligned}
$$

We can now sequentially apply 55 to relate $X$ and $Y$ : Consider

$$
\begin{aligned}
Y_{s}= & \sigma^{(1)} \zeta^{s}\left(\mathbb{1}^{\otimes(2 s+1)} \otimes v^{\otimes(k-1-s)} \otimes \mathbb{1}\right) \times \\
& \left(u^{\dagger \otimes s} \otimes x^{\dagger} \otimes u^{\otimes(k-1-s)}\right)\left(\mathbb{1} \otimes v^{\dagger \otimes s} \otimes \mathbb{1}^{\otimes(2 k-2 s-1)}\right)
\end{aligned}
$$

for $s=0, \ldots, k-1$, and note that by applying (55), we can show that $Y_{s}=Y_{s+1}$. We thus have that

$$
Y=Y_{0}=\cdots=Y_{k-1}=\sigma^{(1)} \zeta^{k-1} X^{\dagger}
$$

and thus $\sigma^{(k)}=\sigma^{(1)} \zeta^{k-1}$ as claimed.

Corollary VI.11. Consider two $M P U \mathcal{U}_{1}$ and $\mathcal{U}_{2}$ in $S F$. For $\mathcal{U}_{1}$ and $\mathcal{U}_{2}$ to be strictly equivalent, it is necessary that both $\sigma^{(1)}$ and $\sigma^{(2)}$ (cf. Prop. VI.10) for the two MPU are equal. For $\mathcal{U}_{1}$ and $\mathcal{U}_{2}$ to be equivalent, it is necessary that $\sigma^{(2)}$ for the two MPU is equal. 


\section{Connection to the classification of phases of Matrix Product States}

Two MPUs are equivalent if they can be connected by a continuous path of MPUs. A necessary condition for the existence of such a path is the existence of a continuous path of normal MPVs, cf. Prop. III.1, without requiring unitarity. This is equivalent to asking whether the corresponding injective MPS are in the same phase as ground states of 1D Hamiltonians. This question has been studied previously 12 14, and it has been found that this relates to the classification of the gauge transformations

$$
\mathcal{S}[\mathcal{U}] \propto G \mathcal{U} G^{-1}
$$

which represent the symmetry action locally [12 14. In particular, for the three cases considered in this paper, it can be easily checked that the classification of $G$ is as follows:

1. Conjugation: $G \bar{G}= \pm \mathbb{1}$.

2. Time reversal: $G \bar{G}= \pm \mathbb{1}$.

3. Transposition: No nontrivial phases.

Since $G$ is invariant under blocking and adding ancillas, it gives necessary conditions for equivalence of MPUs, cf. Def. VI.2. The symmetry in the two first cases corresponds to time reversal symmetry in the MPS classification [12], where the condition is obtained by applying $\mathcal{S}[\mathcal{S}[\mathcal{U}]] \propto \mathcal{U}$. Note that by a suitable choice of gauge, $G$ can be chosen unitary [15, and thus the cases $G \bar{G}= \pm \mathbb{1}$ correspond to symmetric and skew-symmetric unitary matrices, which form two disconnected equivalence classes (cf. Corollary V.3). The transposition symmetry corresponds to an on-site $\mathbb{Z}_{2}$ symmetry, which has no non-trivial projective representations and thus no nontrivial MPS phases [13, 14].

Proposition VI.12. For an $M P U$ invariant under conjugation symmetry, the sign $G \bar{G}= \pm 1$ in the MPS classification of phases, Eq. (56), corresponds to the two cases in Prop. V.3, i.e., the two possible MPU phases under equivalence.

Proof. The statement follows immediately from the fact that the two cases in Prop.V.3 label the two phases under equivalence under conjugation (Corollary VI.7), together with the fact that equality of the $\operatorname{sign} G G= \pm \mathbb{1}$ is a necessary condition for being in the same phase. The fact that Case I corresponds to $G \bar{G}=+\mathbb{1}$ can be checked immediately by setting $u=v=\mathbb{1}$.

Proposition VI.13. For an $M P U$ in SF invariant under time reversal, the sign $G \bar{G}= \pm \mathbb{1}$ in the MPS classification of phases, Eq. (56), corresponds to the sign obtained in Prop. V.5 after blocking $k=2$ sites, i.e., $\sigma^{(2)}$ in Prop. VI.10,
Proof. Consider $\mathcal{U}$ in SF. $\mathcal{U}$ is normal and thus an injective MPS tensor, and thus we have

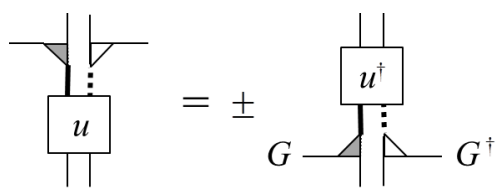

where with an appropriately chosen gauge, $G$ is unitary. Using this, we have that

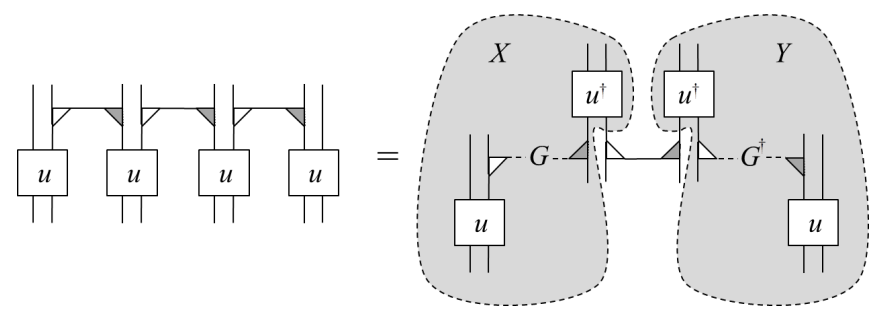

Using that $\bar{G}= \pm G^{\dagger}$, we can now immediately verify that $X^{\dagger}= \pm Y$, and thus, $\sigma^{(2)}= \pm 1$ as claimed.

\section{SYMMETRIES: EXAMPLES}

In this Section, we analyze some relevant examples. First, we give an example for each of the phases that have been identified in the previous section. Then, we consider two MPUs that represent toy models of topological insulators, and study if they are connected among them and to the identity operator under different symmetries.

\section{A. Conjugation symmetry: Completeness of classification}

Let us start by providing examples for all possible phases under conjugation.

Example VII.1 (All phases under conjugation).

1. Let $u_{+}=v_{+}=\mathbb{1}_{r \ell}$, and $\left(u_{-}\right)=\left(v_{-}\right)=$ $\operatorname{diag}(-1,1, \ldots, 1)$. Then,

- in case at least one of $r, \ell$ is odd, the MPUs given by $\left(u_{+}, v_{+}\right)$and by $\left(u_{+}, v_{-}\right)$lie in the two classes of Theorem VI.4.

- in case both $r$ and $\ell$ are even, the MPUs given by $\left(u_{ \pm}, v_{ \pm}\right)$(with all four sign choices) lie in the four classes of Theorem VI.6 for which $u$ and $v$ fall into Case I of Prop. V.3.

2. Let $u_{ \pm}^{\prime}=\left(\mathbb{1}_{r \ell / 4} \otimes R\right) u_{ \pm}$and $v_{ \pm}^{\prime}=\left(\mathbb{1}_{r \ell / 4} \otimes R\right) v_{ \pm}$, with $R=\frac{1-i}{2}\left[\mathbb{1}_{4}+i(Y \otimes Y)\right]$, as in Corollary $V .4$. Then, the MPUs given by $\left(u_{ \pm}^{\prime}, v_{ \pm}^{\prime}\right)$ (with all four sign choices) lie in the four classes of Theorem VI.6 for which $u$ and $v$ fall into Case II of Prop. V.3. 
The verification is straightforward by using that $u_{ \pm}, v_{ \pm}$ are unitary, $u_{ \pm}^{\prime}, v_{ \pm}^{\prime}$ fall into Case II following Corol-

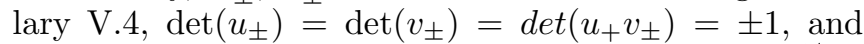
$\operatorname{det}(R)=-1$ and thus $\operatorname{det}\left(u_{+}^{\prime}\right)=\operatorname{det}\left(v_{+}^{\prime}\right)= \pm(-1)^{r \ell / 4}$.

Note that e.g. for the MPU described by $u=v=R$, the corresponding $\mathcal{U}$ is

$$
\mathcal{U}_{\ell r}^{u d}=\frac{1-i}{2}\left(\left(\sigma_{y}\right)^{\ell}\left(-i \sigma_{y}\right)^{r}\right)_{u d}
$$

with $\ell, r, u, d=0,1$ denoting the left, right, up, and down indices, respectively. This $\mathcal{U}$ needs to be blocked once to obtain the SF; then, it is straightforward to check that conjugation maps to a $G=\sigma_{y}$ gauge, which satisfies $G \bar{G}=-\mathbb{1}$, i.e., we are in the non-trivial MPS phase (as shown in Prop. VI.12. It can be easily seen that this generalized to all above examples, as the virtual degrees of freedom relating to $u_{ \pm}$and $v_{ \pm}$transform trivially under conjugation: Their MPU tensor is given by $\delta_{u=d=\ell} x_{\ell r}$ with $x=u_{ \pm}, v_{ \pm}$and thus real.

Let us also briefly consider these examples in the light of the other symmetries. Clearly, the MPUs given by $\left(u_{ \pm}, v_{ \pm}\right)$have time reversal and transposition symmetry, and lie in the trivial class with regard to the former. In the second case, it is easy to check that only the MPU with $\left(u_{+}^{\prime}, v_{+}^{\prime}\right)$ has time reversal and transposition symmetry (the others violate it already on two sites); the latter has $\sigma^{(1)}=\sigma^{(2)}=1$ in Prop. VI.10.

\section{B. Time-reversal symmetry}

Let us now provide examples for the four classes which we identified under time reversal symmetry, namely $\sigma^{(1)}= \pm 1$ and $\sigma^{(2)}= \pm 1$, Prop. VI.10.

Example VII.2 (CZX). The CZX MPU [10] is defined by

$$
\begin{aligned}
& u=C Z\left(\sigma_{x} \otimes \sigma_{x}\right) \\
& v=C Z
\end{aligned}
$$

where $C Z=\operatorname{diag}(1,1,1,-1)$ is the controlled- $Z$ gate. The corresponding $\mathcal{U}$ is

$$
\mathcal{U}_{\ell r}^{u d}=\left(\sigma_{x}\right)_{u d} \delta_{u, \ell} H_{\ell, r}
$$

with $\ell, r, u, d=0,1$ denoting the left, right, up, and down indices, respectively, and $H=\frac{1}{\sqrt{2}}\left(\begin{array}{ll}1 & 1 \\ 1 & -1\end{array}\right)$ the Hadamard gate.

It is straightforward to check that this example has $\sigma^{(1)}=-1$ and $\sigma^{(2)}=-1$ in Prop. VI.10. By blocking two sites of the MPS description, we obtain SF (and thus injectivity), and we find that the virtual gauge transformation arising from time reversal is $G=\sigma_{y}$, corresponding to the non-trivial phase (as shown in Prop. VI.13).

Note that this example also has the other two symmetries. For conjugation symmetry and strict equivalence, it is in the class which satisfies Case I and has $\operatorname{det}(u)=\operatorname{det}(v)=-1$ in Theorem VI.6. Under blocking, it thus falls into the trivial class, which can also be verified using the MPS representation (the gauge is $G=\mathbb{1}$ ).

The trivial case under time reversal can just be realized by setting $u=v=\mathbb{1}$. The other non-trivial cases of $\sigma^{(1)}$ and $\sigma^{(2)}$ can be constructed by modifying the trivial and CZX example, respectively.

Example VII.3. 1. The $M P U u=v=\mathbb{1}$ realizes the trivial case $\sigma^{(1)}=\sigma^{(2)}=1$.

2. The modified trivial $M P U u=i \mathbb{1}, v=\mathbb{1}$ realizes the case $\sigma^{(1)}=-1, \sigma^{(2)}=1$.

3. The modified $C Z X M P U u=i C Z\left(\sigma_{x} \otimes \sigma_{x}\right), v=C Z$ realizes the case $\sigma^{(1)}=1, \sigma^{(2)}=-1$.

Finally, these examples can be extended to any even dimension $\ell=r=d$ by tensoring the CZX example with $\mathbb{1}_{d / 2}$; clearly, the argument of ExampleVII.3 still applies.

\section{Examples motivated by topological insulators}

In the following, we will discuss a class of examples motivated by topological insulators, where the edge currents for each component of the spin are chiral. Thus, a toy model describing the dynamics of the edge states would correspond to a spin $1 / 2$ particle; if it is up, it moves to the right and if it is down, to the left. Here we will consider many-body versions of such model.

\section{The Examples}

We consider here three examples where the local dimension is $\tilde{d}=d^{2}$, i.e. it corresponds to having two spins per site. We define the shift operator [1], $T^{(N)}$, acting on $H_{d}^{\otimes N}$ as

$$
T^{(N)}\left|n_{1}, \ldots, n_{N}\right\rangle=\left|n_{N}, n_{1}, \ldots, n_{N-1}\right\rangle .
$$

That is, it shifts product states to the right. Its adjoint, $T^{(N) \dagger}$ shifts to the left. We also define

$$
\begin{aligned}
& U_{1}^{(N)}=(\mathbb{1} \otimes \mathbb{1})^{\otimes N}, \\
& U_{2}^{(N)}=T^{(N) \dagger} \otimes T^{(N)}, \\
& U_{3}^{(N)}=T^{(N)} \otimes T^{(N) \dagger} .
\end{aligned}
$$

The physical meaning of the second (third) unitaries is simple: In a site, the spin on the right is shifted to the right (left) and the one on the left, to the left (right). We illustrate $U_{2}^{(N)}$ and $U_{3}^{(N)}$ in Fig. 1. Note that $U_{3}^{(N)}=$ $\mathbb{S}^{\otimes N} U_{2}^{(N)} \mathbb{S}^{\otimes N}$.

The matrices $u_{i}, v_{i}(i=1,2,3)$ generating $U_{i}^{(N)}$ can be easily derived from Fig. 1. We have

$$
\begin{array}{ll}
u_{1}=\mathbb{1} \otimes \mathbb{1}, & v_{1}=\mathbb{1} \otimes \mathbb{1}, \\
u_{3}=\mathbb{S}, & v_{3}=\mathbb{S} .
\end{array}
$$


a)

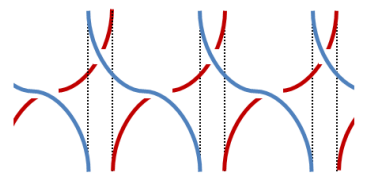

b)

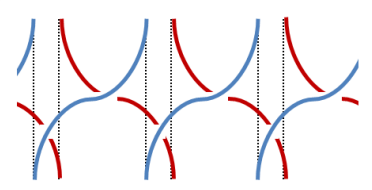

FIG. 1. Graphical representation of: a) $U_{2}^{(N)}$, and b) $U_{3}^{(N)}$. Solid lines indicate how different spins are shifted.

For the ones corresponding to $U_{2}^{(N)}$ we must block two sites, which yields the blocked matrices

$$
\begin{aligned}
& u_{2}^{(2)}=\mathbb{1} \otimes \mathbb{S} \otimes \mathbb{1}, \\
& v_{2}^{(2)}=(\mathbb{S} \otimes \mathbb{S})(\mathbb{1} \otimes \mathbb{S} \otimes \mathbb{1}) .
\end{aligned}
$$

and correspondingly

$$
\begin{aligned}
& u_{3}^{(2)}=(\mathbb{1} \otimes \mathbb{S} \otimes \mathbb{1})(\mathbb{S} \otimes \mathbb{S}), \\
& v_{3}^{(2)}=(\mathbb{1} \otimes \mathbb{S} \otimes \mathbb{1}),
\end{aligned}
$$

All three MPUs in Eq. (58) are generated by tensors which have ind $=0$ (this follows from Theorem IV.6 and the fact that $T^{(N)}$ has index ind $=\log _{2}\left(d_{0}\right)$ whereas $T^{(N) \dagger}$ has ind $\left.=-\log _{2}\left(d_{0}\right)\right)$. They have conjugation symmetry, as well as the symmetries 490 with $Q=\mathbb{S}$, this is, time reversal or transpose combined with an exchanging the two species of sites.

\section{Transformation with Swaps}

In the following we will analyze whether the three MPU in Eq. (58) are in the same phase with respect to the three symmetries mentioned above, this is, conjugation as well as the symmetries of Eq. (49) with $Q=\mathbb{S}$. In the latter case, following Section VD, it is convenient to define new MPU, $\tilde{\mathcal{U}}=\mathcal{U} \mathbb{S}$, generating

$$
\begin{aligned}
& \tilde{U}_{1}^{(N)}=\mathbb{S}^{\otimes N}, \\
& \tilde{U}_{2}^{(N)}=\mathbb{S}^{\otimes N}\left(T^{(N) \dagger} \otimes T^{(N)}\right), \\
& \tilde{U}_{3}^{(N)}=\mathbb{S}^{\otimes N}\left(T^{(N)} \otimes T^{(N) \dagger}\right)=\mathbb{S}^{\otimes N} \tilde{U}_{2}^{(N)} \mathbb{S}^{\otimes N} .
\end{aligned}
$$

Lemma VII.4. Any two $M P U U_{i}^{(N)}$ and $U_{j}^{(N)}$ in (58) are in the same phase under (49a), 49b) (both with $Q=\mathbb{S})$, and conjugation, respectively, iff the corresponding $M P U \tilde{U}_{i}^{(N)}$ and $\tilde{U}_{j}^{(N)}$ in (58) are in the same phase under time reversal, transposition, and conjugation, respectively.

Proof. The first two symmetries follow from the discussion in Sec. VD, The last follows from the fact that $\tilde{U}^{(N)}=\mathbb{S}^{\otimes N}$ is invariant under conjugation iff $U^{(N)}$ is, together with the the fact that any continuous path
$U(x)^{(N)}$ with conjugation symmetry gives rise to a continuous path $\tilde{U}(x)^{(N)}=\mathbb{S}^{\otimes N} U(x)^{(N)} \mathbb{S}^{\otimes N}$ with conjugation symmetry, and vice versa. Note that in all cases, translation symmetry is kept (though the transformation might break or restore the SF).

Thus, for analyzing those symmetries we can restrict ourselves to the form 62.

The SF of tensors generating the MPUs $\tilde{U}_{1}^{(N)}$ and $\tilde{U}_{2}^{(N)}$ have

$$
\begin{aligned}
& \tilde{u}_{1}=\mathbb{S}, \quad \tilde{v}_{1}=\mathbb{1} \otimes \mathbb{1}, \\
& \tilde{u}_{2}=\mathbb{1} \otimes \mathbb{1}, \quad \tilde{v}_{2}=\mathbb{S} .
\end{aligned}
$$

(We will not need the one corresponding to the third.) Note that all three MPUs have the same index and spectrum, and thus just by looking at spectral properties we cannot distinguish their phases under time reversal (as the spectrum must be real and thus cannot be changed continuously). The fact that $\tilde{U}_{2}^{(N)}$ and $\tilde{U}_{3}^{(N)}$ have the same spectrum is clear since they are related by a unitary transformation, the global swap $(62 \mathrm{c})$. The fact that $\tilde{U}_{1}^{(N)}$ and $\tilde{U}_{2}^{(N)}$ have the same spectrum is obvious from their SF 63 .

$$
\text { 3. Phases of } U_{2}^{(N)} \text { vs. } U_{3}^{(N)}
$$

Let us now compare $U_{2}^{(N)}$ vs. $U_{3}^{(N)}$ under the three symmetries. We start with conjugation. In order to compare the two, we have to block two sites and thus consider the SF given in Eqs. 60 and 61), respectively. First, since $u_{i}^{(2)}, v_{i}^{(2)}$ are real, they transform trivally under conjugation and we are thus in Case I. We then have that

$$
\begin{aligned}
& \operatorname{det}\left(u_{2}^{(2)}\right)=\operatorname{det}\left(v_{3}^{(2)}\right)=\operatorname{det}(\mathbb{S})^{d^{2}} \\
& \operatorname{det}\left(u_{3}^{(2)}\right)=\operatorname{det}\left(v_{2}^{(2)}\right)=\left(\operatorname{det}(\mathbb{S})^{d^{2}}\right)^{3}=\operatorname{det}(\mathbb{S})^{d^{2}},
\end{aligned}
$$

where we have used that $\operatorname{det}(\mathbb{S})= \pm 1$. If $d$ is odd, Theorem VI.4 applies and $\operatorname{det}\left(u_{2}^{(2)}\right)=\operatorname{det}\left(v_{2}^{(2)}\right)$ and thus $\operatorname{det}\left(u_{2}^{(2)} v_{2}^{(2)}\right)=1$; if $d$ is even, Theorem VI.6 applies with $\operatorname{det}\left(u_{i}^{(2)}\right)=\operatorname{det}\left(v_{i}^{(2)}\right)=1$. We thus find:

Proposition VII.5. After blocking 2 sites, $U_{2}^{(N)}$ and $U_{3}^{(N)}$ are both in the trivial phase according to conjugation.

Now, let us move to the other symmetries.

Proposition VII.6. The $M P U \tilde{U}_{2,3}^{(N)}$ are in the same phase with respect to both time reversal and transposition symmetry.

Proof. We will show that there exists a continuous path of tensors $\mathcal{U}(x), x \in[0,1]$, generating $\tilde{U}(x)^{(N)}$, such that $\tilde{U}(0)^{(N)}=\tilde{U}_{2}^{(N)}$ and $\tilde{U}(1)^{(N)}=\tilde{U}_{3}^{(N)}$. Let us denote 
by $\mathcal{U}$ the tensor generating $\tilde{U}_{2}^{(N)}$. We choose a unitary operator $\mathbb{S}(x)$ such that $\mathbb{S}(0)=\mathbb{1}$ and $\mathbb{S}(1)=\mathbb{S}$, and define $\mathcal{U}(x)=\mathbb{S}(x) \mathcal{U} \mathbb{S}(x)^{\dagger}$, where $\mathbb{S}(x)$ acts on the physical indices of $\mathcal{U}$. It is obvious that this generates a self-adjoint MPU. Together with (62c), this yields the statement of the proposition. A similar construction with $\mathbb{S}(x)^{\dagger} \rightarrow \mathbb{S}(x)^{T}$ works for the transposition symmetry.

\section{Phases of $U_{1}^{(N)}$ vs. $U_{2}^{(N)}$}

We now turn towards $U_{1}^{(N)}$ vs. $U_{2}^{(N)}$. Let us start with conjugation symmetry.

Proposition VII.7. $U_{1}^{(N)}$ and $U_{2}^{(N)}$ are in different phases if $d=4 k+2, k=0,1,2, \ldots$, and otherwise in the same phase, where the phase of $U_{1}^{(N)}$ is (by construction) trivial. All phases are in Case $I$, and thus $U_{1}^{(N)}$ and $U_{2}^{(N)}$ coincide under equivalence (i.e. blocking and adding ancillas).

Proof. Following Lemma VII.4 we can consider $\tilde{U}_{1}^{(N)}$ and $\tilde{U}_{2}^{(N)}$ instead, and consider the SF 63 . Again, both fall into Case I. We have that

$$
\operatorname{det}\left(\tilde{u}_{1}\right)=\operatorname{det}\left(\tilde{v}_{2}\right)=\operatorname{det}(\mathbb{S})=(-1)^{d(d-1) / 2},
$$

while $\operatorname{det}\left(\tilde{v}_{1}\right)=\operatorname{det}\left(\tilde{u}_{2}\right)=1$. Thus, for $d$ odd, $\operatorname{det}\left(\tilde{u}_{1}\right) \operatorname{det}\left(\tilde{v}_{1}\right)=\operatorname{det}\left(\tilde{u}_{2}\right) \operatorname{det}\left(\tilde{v}_{2}\right)=(-1)^{d(d-1) / 2}$ and thus $\tilde{U}_{1}^{(N)}$ and $\tilde{U}_{2}^{(N)}$ are in the same phase (Theorem VI.4); it is non-trivial if $d=4 k+3$. If $d$ is even, $\operatorname{det}\left(\tilde{u}_{1}\right)=\operatorname{det}\left(\tilde{v}_{1}\right)=1$ and $1=\operatorname{det}\left(\tilde{u}_{2}\right)=\operatorname{det}\left(\tilde{v}_{2}\right)$ iff $d(d-1) / 2$ is even, this is $d$ is divisible by $4: \tilde{U}_{1}^{(N)}$ and $\tilde{U}_{2}^{(N)}$ are in the same phase if $d=4 k$, and in different phases if $d=4 k+2$ (Theorem VI.6).

As already indicated in the proof, for $d=4 k+2$ the two phases of $\tilde{U}_{1}^{(N)}$ and $\tilde{U}_{2}^{(N)}$ are both non-trivial, while in the other cases, the joint phase which both MPUs share is only non-trival if $d=4 k+3$. Since we did not block here, we in principle cannot translate this back to a statement about the nature of the phase of $U_{2}^{(2)}$. However, since $U_{1}^{(N)}$ is by construction in the trivial phase, and we know how $U_{2}^{(N)}$ relates to $U_{1}^{(N)}$, this nevertheless allows to infer whether the phase of $U_{2}^{(N)}$ is non-trivial.

Let us now turn towards the other two symmetries. It can be checked straightforwardly from 63 that $\sigma^{(1)}=$ $\sigma^{(2)}=1$ in Prop. VI.10, i.e., we obtain no separation of $\tilde{U}_{1}^{(N)}$ and $\tilde{U}_{2}^{(N)}$ under time reversal. Indeed, it is possible to show that $\tilde{U}_{1}^{(N)}$ and $\tilde{U}_{2}^{(N)}$ are in the same phase once we allow for ancillas:

Proposition VII.8. If we add one ancilla of dimension $d$ per site, $\tilde{U}_{1}^{(N)}$ and $\tilde{U}_{2}^{(N)}$ are stricly equivalent both under time reversal and transposition symmetry.

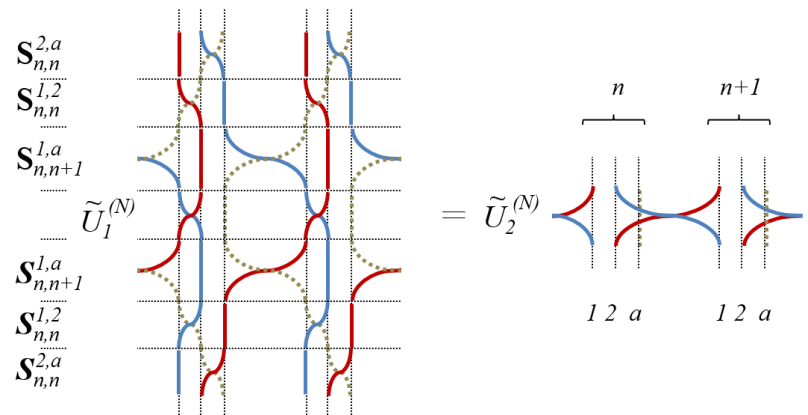

FIG. 2. Proof of Prop. VII.8 By adding an ancilla, $\tilde{U}_{1}^{(N)}$ and $\tilde{U}_{2}^{(N)}$ can be connected through a path with time reversal or transposition symmetry.

Proof. The proof is illustrated in Fig. 2. By adding an ancillas and conjugating $\tilde{U}_{1}^{(N)}$ with the three swaps shown, we obtain $\tilde{U}_{2}^{(N)}$. We can now choose a continuous interpolation $\mathbb{S}(x)$ such that $\mathbb{S}(0)=\mathbb{1}$ and $\mathbb{S}(1)=\mathbb{S}$. By conjugating with $\mathbb{S}(x)$ and $\mathbb{S}(x)^{\dagger}$ or $\mathbb{S}(x)^{T}$ instead of the swaps, we obtain a continuous time reversal or transposition symmetric path which connects $\tilde{U}_{1}(N)$ and $\tilde{U}_{2}(N)$, as claimed.

$$
\text { 5. Phases of } U_{1}^{(N)} \text { vs. } U_{3}^{(N)}
$$

Finally, let us consider the phase of $U_{1}^{(N)}$ vs. $U_{3}^{(N)}$. It is immediate to see that all results from the preceding section can be transferred, e.g. by observing that there is no relevance to the ordering of the two spins per site, and swapping them exchanges $U_{2}^{(N)}$ with $U_{3}^{(N)}$ (and $\tilde{U}_{2}^{(N)}$ with $\left.\tilde{U}_{3}^{(N)}\right)$, or by noting the correspondence between Eqs. (59) and (63). Note that in this case, however, the proof analogous to that of Prop. VII.7 will additionally allow to determine the phase of $U_{2}^{(N)}$ under strict equivalence, i.e., without any blocking. (If one is only interested in the results after blocking two sites, one can alternatively also transfer the results of the preceding section from $U_{2}^{(N)}$ to $U_{3}^{(N)}$ using Propositions VII.5 and VII.6.)

\section{ACKNOWLEDGMENTS}

This project has been supported by the EU through the ERC grants WASCOSYS (No. 636201), GAPS (No. 648913), and QUTE (No. 647905). DPG acknowledges support by MINECO through grants MTM201454240-P and ICMAT Severo Ochoa SEV-2015-0554, and by Comunidad de Madrid through grant QUITEMAD+CM, ref. S2013/ICE-2801. 


\section{Appendix A: MPU and QCA}

In this appendix we recall from [16] the definition of QCA and prove that 1D QCAs and MPUs are exactly the same objects. We also show that the index defined here coincides (up to a $\log$ ) with the original index of QCA introduced in Ref. 11.

In order to define QCA we have to start with the quasilocal algebra of observables in a 1D spin chain. Let us take a finite $\Lambda \subset \mathbb{Z}$ and denote by $\mathcal{A}_{\Lambda}$ the algebra of observables of $\Lambda$, that is $M_{d}^{\otimes \Lambda}$. If $\Lambda \subset \Lambda^{\prime}$ one can identify $\mathcal{A}_{\Lambda}$ as a subset of $\mathcal{A}_{\Lambda^{\prime}}$ by tensoring each element with the identity in the sites of $\Lambda^{\prime} \backslash \Lambda$. This allows to define the inductive limit

$$
\mathcal{A}_{\mathrm{loc}}=\bigcup_{\Lambda}^{\infty} \mathcal{A}_{\Lambda}
$$

After completion with the norm topology one obtains a $C^{*}$-algebra $\mathcal{A}$ called the quasi-local algebra of observables.

A 1D QCA is simply an automorphism $\omega$ of $\mathcal{A}$, which commutes with the translation operator and for which there exists a finite subset $\mathcal{N} \subset \mathbb{Z}$ so that $\omega\left(\mathcal{A}_{\Lambda}\right) \subset \mathcal{A}_{\Lambda+\mathcal{N}}$ for all finite $\Lambda \subset \mathbb{Z}$. Wlog one can assume that $\mathcal{N}$ is an interval $[-R, R] \cap \mathbb{Z}$.

Let us show first that any MPU given by tensor $\mathcal{U}$ defines a QCA. For that we consider $\omega_{\mathcal{U}}: \mathcal{A}_{\text {loc }} \rightarrow \mathcal{A}_{\text {loc }}$ given by

$$
\omega_{\mathcal{U}}(A)=\lim _{N} U^{(N)} A_{L} U^{(N) \dagger} .
$$

Note that $\omega_{\mathcal{U}}$ is well defined since the limit is eventually constant thanks to the results proven in the main text. Moreover, $\omega_{\mathcal{U}}$ preserves the norm and all opera- tions of the *-algebra $\mathcal{A}_{\text {loc }}$, commutes with the translation operator and verifies that $\omega_{\mathcal{U}}\left(\mathcal{A}_{\Lambda}\right) \subset \mathcal{A}_{\Lambda+\mathcal{N}}$ for $\mathcal{N}=\left[-4 D^{4}, 4 D^{4}\right] \cap \mathbb{Z}$. It can be then uniquely extended to a $1 \mathrm{D}$ QCA as defined above.

Conversely, by the main result of [16, after blocking a finite number of sites, any $1 \mathrm{D}$ QCA $\omega$ restricted to $\mathcal{A}_{\text {loc }}$ is exactly of the form $(\mathrm{A} 2)$, where the unitaries $U^{(N)}$ are given by depth-two circuits of nearest neighbor unitaries $u$ and $v$. This gives exactly the standard form (SF) of an MPU $\mathcal{U}(33)$ and $\omega=\omega_{\mathcal{U}}$ for such MPU.

Let us finish this appendix by showing that the index defined here coincides with the one defined in [1]. We will use the identification just made between QCA and MPU. Let us recall the original definition of 1 . For that we group sites together so that the MPU $\mathcal{U}$ is simple. Then clearly, for $x \in \mathbb{Z}$,

$$
\omega\left(\mathcal{A}_{2 x} \otimes \mathcal{A}_{2 x+1}\right) \subset\left(\mathcal{A}_{2 x-1} \otimes \mathcal{A}_{2 x}\right) \otimes\left(\mathcal{A}_{2 x+1} \otimes \mathcal{A}_{2 x+2}\right) .
$$

Note that all algebras $\mathcal{A}_{z}, z \in \mathbb{Z}$, are isomorphic to $M_{d}$.

In order to define the index, one needs to identify the support algebra of $\omega\left(\mathcal{A}_{2 x} \otimes \mathcal{A}_{2 x+1}\right)$ on $\mathcal{A}_{2 x-1} \otimes \mathcal{A}_{2 x}$, that is, the smallest $C^{*}$-subalgebra $\mathcal{R}_{2 x}$ of $\mathcal{A}_{2 x-1} \otimes \mathcal{A}_{2 x}$ so that $\omega\left(\mathcal{A}_{2 x} \otimes \mathcal{A}_{2 x+1}\right) \subset \mathcal{R}_{2 x} \otimes\left(\mathcal{A}_{2 x+1} \otimes \mathcal{A}_{2 x+2}\right)$. It is shown in [1] that $\mathcal{R}_{2 x}$ is isomorphic to a matrix algebra $M_{s}$ and the index is defined as

$$
\text { Original index }=\frac{s}{d} .
$$

It is clear from the standard form of the MPU (33) that $\mathcal{R}_{2 x}=v^{\dagger}\left(\mathbb{1} \otimes M_{\ell}\right) v$ and hence

$$
\text { ind } \left.=-2 \log _{2} \frac{\ell}{d}=-2 \log _{2} \text { (Original index }\right) \text {. }
$$

[1] D. Gross, V. Nesme, H. Vogts, and R. F. Werner, Commun. Math. Phys. 310, 419 (2012), arXiv:0910.3675.

[2] M. Hastings, J. Stat. Mech. , P08024 (2007), arXiv:0705.2024,

[3] F. Verstraete and J. I. Cirac, Phys. Rev. B 73, 094423 (2006), cond-mat/0505140.

[4] F. Verstraete, J. J. Garcia-Ripoll, and J. I. Cirac, Phys. Rev. Lett. 93, 207204 (2004), cond-mat/0406426

[5] M. Zwolak and G. Vidal, Phys. Rev. Lett. 93, 207205 (2004), cond-mat/0406440.

[6] J. I. Cirac, D. Poilblanc, N. Schuch, and F. Verstraete, Phys. Rev. B 83, 245134 (2011), arXiv:1103.3427.

[7] M. B. Sahinoglu et al., (2014), arXiv:1409.2150

[8] N. Bultinck et al., (2015), arXiv:1511.08090

[9] See, e.g., H. C. Po, L. Fidkowski, T. Morimoto, A. C. Potter, and A. Vishwanath, Phys. Rev. X 6, 041070 (2016), arXiv:1609.00006, and references therein.

[10] X. Chen, Z.-X. Liu, and X.-G. Wen, Phys. Rev. B 84, 235141 (2011), arXiv:1106.4752.

[11] D. Perez-Garcia, F. Verstraete, M. M. Wolf, and J. I. Cirac, Quant. Inf. Comput. 7, 401 (2007), quant-

\section{$\mathrm{ph} / 0608197$.}

[12] F. Pollmann, A. M. Turner, E. Berg, and M. Oshikawa, Phys. Rev. B 81, 064439 (2010), arXiv:0910.1811.

[13] X. Chen, Z. Gu, and X. Wen, Phys. Rev. B 83, 035107 (2011), arXiv:1008.3745

[14] N. Schuch, D. Perez-Garcia, and I. Cirac, Phys. Rev. B 84, 165139 (2011), arXiv:1010.3732.

[15] J. Cirac, D. Perez-Garcia, N. Schuch, and F. Verstraete, Ann. Phys. 378, 100 (2017), arXiv:1606.00608.

[16] B. Schumacher and R. F. Werner, quant-ph/0405174

[17] C. Cedzich et al., arXiv:1611.04439

[18] L. Fidkowski, H. C. Po, A. C. Potter, and A. Vishwanath, (2017), arXiv:1703.07360.

[19] M. Nagata, J. Math. Soc. Japan 4, 296 (1952).

[20] G. Higman, Proceedings of the Cambridge Philosophical Society 52, 1 (1956).

[21] Y. Razmyslov, Trace identities of full matrix algebras over a field of characteristic zero (in Russian). Izv. Akad. Nauk SSSR, Ser. Mat., 38 (1974), 723-956. (English translation: Math. USSR, Izv., 8 (1974), 727-760 (1975)).

[22] E. I. Zelmanov, On the nilpotency of nil algebras, Al- 
gebra - some current trends (Varna, 1986), pg. 227-240. Lecture Notes in Math. 1352. Springer, Berlin 1988.

[23] D. Montgomery and C. T. Yang, Ann. Math. 65, 108 (1957).
[24] G. E. Brendon, Introduction to compact transformation groups (Academic Press, 1972).

[25] M. Sanz, M. M. Wolf, D. Perez-Garcia, and J. I. Cirac, Phys. Rev. A 79, 042308 (2009), arXiv:0901.2223 